\title{
Near-Optimal Learning of Tree-Structured Distributions by Chow-Liu
}

\author{
Arnab Bhattacharyya \\ National University of Singapore \\ Singapore \\ arnabb@nus.edu.sg \\ Eric Price \\ University of Texas at Austin \\ Austin, USA \\ ecprice@cs.utexas.edu
}

\author{
Sutanu Gayen \\ National University of Singapore \\ Singapore \\ sutanugayen@gmail.com \\ N. V. Vinodchandran \\ University of Nebraska-Lincoln \\ Lincoln, USA \\ vinod@cse.unl.edu
}

\begin{abstract}
We provide finite sample guarantees for the classical Chow-Liu algorithm (IEEE Trans. Inform. Theory, 1968) to learn a tree-structured graphical model of a distribution. For a distribution $P$ on $\Sigma^{n}$ and a tree $T$ on $n$ nodes, we say $T$ is an $\varepsilon$-approximate tree for $P$ if there is a $T$-structured distribution $Q$ such that $D(P \| Q)$ is at most $\varepsilon$ more than the best possible tree-structured distribution for $P$. We show that if $P$ itself is tree-structured, then the ChowLiu algorithm with the plug-in estimator for mutual information with $\widetilde{O}\left(|\Sigma|^{3} n \varepsilon^{-1}\right)$ i.i.d. samples outputs an $\varepsilon$-approximate tree for $P$ with constant probability. In contrast, for a general $P$ (which may not be tree-structured), $\Omega\left(n^{2} \varepsilon^{-2}\right)$ samples are necessary to find an $\varepsilon$-approximate tree. Our upper bound is based on a new conditional independence tester that addresses an open problem posed by Canonne, Diakonikolas, Kane, and Stewart (STOC, 2018): we prove that for three random variables $X, Y, Z$ each over $\Sigma$, testing if $I(X ; Y \mid Z)$ is 0 or $\geq \varepsilon$ is possible with $\widetilde{O}\left(|\Sigma|^{3} / \varepsilon\right)$ samples. Finally, we show that for a specific tree $T$, with $\widetilde{O}\left(|\Sigma|^{2} n \varepsilon^{-1}\right)$ samples from a distribution $P$ over $\Sigma^{n}$, one can efficiently learn the closest $T$-structured distribution in KL divergence by applying the add-1 estimator at each node.
\end{abstract}

\section{CCS CONCEPTS}

- Mathematics of computing $\rightarrow$ Information theory; Multivariate statistics; Hypothesis testing and confidence interval computation; Maximum likelihood estimation.

\section{KEYWORDS}

Chow Liu tree, learning theory, high-dimensional statistics, distribution testing

\section{ACM Reference Format:}

Arnab Bhattacharyya, Sutanu Gayen, Eric Price, and N. V. Vinodchandran. 2021. Near-Optimal Learning of Tree-Structured Distributions by Chow-Liu. In Proceedings of the 53rd Annual ACM SIGACT Symposium on Theory of

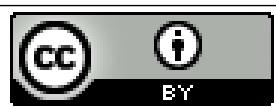

This work is licensed under a Creative Commons Attribution International 4.0 License. STOC '21, June 21-25, 2021, Virtual, Italy

(C) 2021 Copyright held by the owner/author(s).

ACM ISBN 978-1-4503-8053-9/21/06.

https://doi.org/10.1145/3406325.3451066
Computing (STOC '21), fune 21-25, 2021, Virtual, Italy. ACM, New York, NY, USA, 14 pages. https://doi.org/10.1145/3406325.3451066

\section{INTRODUCTION}

Probabilistic graphical models form a highly effective framework for encoding high-dimensional distributions. Graphical models yield human-interpretable representation of data as they explicitly describe the statistical dependencies among different features. From a computational standpoint, the graphical representation enables efficient algorithms for inference, e.g., message passing, loopy belief propagation, and other variational inference methods [40]. Graphical models have found extensive applications in many domains, such as image processing, natural language processing and computational biology; see $[39,42,56]$ and the references therein for examples.

A fundamental question in this area is to learn graphical models from independently drawn samples. In this paper, we focus on the basic problem of learning tree-structured distributions. Given a tree $T$ on $n$ nodes, fix an arbitrary root and orient it outwards. A distribution $P$ over variables $X_{1}, \ldots, X_{n}$ is said to be $T$-structured iff for every non-root vertex $i$ :

$$
X_{i}=f_{i}\left(X_{\mathrm{pa}(i)}, U_{i}\right)
$$

where $\mathrm{pa}(i)$ is the parent of $i$ in the oriented tree, $U_{i}$ is an independent random variable, and $f_{i}$ is a (deterministic) function. A distribution is tree-structured if it is $T$-rooted for some tree $T$. Equivalently, a tree-structured distribution is a Markov random field where the underlying undirected graph is a tree.

In a seminal work [19], Chow and Liu observed that the treestructured distribution maximizing the likelihood of the observed samples can be obtained by solving a maximum weight spanning tree problem. In particular, their algorithm assigns a weight equal to the empirical mutual information between each pair of variables and finds a maximum weight spanning tree in this weighted graph. The resulting tree can be oriented from an arbitrary root, so as to assign a parent $\mathrm{pa}(i)$ for all non-root vertices $i$. Finally, the conditional probability distributions $X_{i} \mid X_{\mathrm{pa}(i)}$ can be learned from the data.

Chow and Wagner [18] showed that the Chow-Liu algorithm consistently recovers structure, meaning that if the samples are generated by a $T$-structured distribution for a tree $T$, then it recovers $T$ with probability approaching 1 as the number of samples tends to infinity. More recent works $[51,52]$ have used large-deviation 
theory to study the error exponent $K_{P}$ of $T$-structured distributions $P$, where:

$$
K_{P}=\lim _{N \rightarrow \infty}-\frac{1}{N} \log \operatorname{Pr}[\widehat{T} \neq T] .
$$

Here, $\widehat{T}$ is the tree output by the Chow-Liu algorithm, and $N$ is the number of samples used. The bounds they obtain depend on the distribution structure, since it may be very hard to distinguish $T$ from an alternative tree that is almost as good.

In this work, we take a different viewpoint that is in the spirit of distribution learning and probabilistically approximately correct (PAC) analysis [36, 37, 53]. Instead of trying to exactly recover the structure of a tree-structured distribution $P$, we consider the objective of learning a tree-structured distribution $Q$ that is close to $P$. For many downstream tasks, most notably statistical inference, it is fine to not recover the exact structure as long as one can approximate probabilities of relevant events using the learned distribution. Also, this viewpoint allows us to analyze Chow-Liu for non-tree structured distributions $P$, by comparing how far $P$ is from the output of Chow-Liu and how far from the closest tree-structured distribution.

More formally, for a distribution $P$ over $\Sigma^{n}$ and a tree $T$ on $n$ vertices, let:

$$
P_{T}:=\underset{\substack{T \text {-structured } \\ \text { distribution } Q}}{\arg \min } D(P \| Q)
$$

where $D(\cdot \| \cdot)$ denotes the KL-divergence. We say that a tree $\widehat{T}$ is an $\varepsilon$-approximate tree for $P$ if:

$$
D\left(P \| P_{\widehat{T}}\right) \leq \min _{\text {tree } T} D\left(P \| P_{T}\right)+\varepsilon .
$$

The KL divergence, although not a metric, is a useful notion of distance to consider in this setting. Firstly, with infinite samples, Chow-Liu's output maximizes the likelihood of generating samples from $P$ and hence, minimizes $D(P \| \cdot)$. Secondly, via Pinsker's inequality, bounding the KL divergence by $\varepsilon$ implies a $\sqrt{2 \varepsilon}$ bound on total variation distance which may be more directly useful.

\subsection{Our Contributions}

We study the number of samples required by Chow-Liu to output an $\varepsilon$-approximate tree with a fixed error probability. We first observe that for any distribution $P$, it can be guaranteed that the output of Chow-Liu is $\varepsilon$-approximate if each mutual information estimate is an additive $\pm \frac{\varepsilon}{2 n}$ estimate. Known bounds for the plug-in entropy estimator imply the following sample complexity.

LeMmA 1.1. The Chow-Liu algorithm when run on $\widetilde{O}\left(\frac{|\Sigma|^{2} n}{\varepsilon}+\right.$ $\left.\frac{n^{2}}{\varepsilon^{2}} \log \frac{1}{\delta}\right)$ samples from a distribution $P$ on $\Sigma^{n}$ outputs an $\varepsilon$-approx. tree $T$ with probability at least $1-\delta$. Moreover, the dependence of the sample complexity on $n$ and $\varepsilon$ are tight up to logarithmic factors.

We show that the quadratic dependence on $n$ and $\varepsilon$ is unfortunately necessary for general distributions $P$. However, in the "realizable" setting where $P$ is actually tree-structured, we show that the sample complexity can be improved to near-linear:

Theorem 1.2. The Chow-Liu algorithm when run on $\widetilde{O}\left(\frac{|\Sigma|^{3} n}{\varepsilon} \log \frac{1}{\delta}\right)$ samples from a tree-structured distribution $P$ on $\Sigma^{n}$ outputs an $\varepsilon^{-}$ approximate tree $T$ with probability at least $1-\delta$. Moreover, the dependence on $n$ and $\varepsilon$ are tight up to logarithmic factors.
Hence, for example, for tree-structured Ising models (where $\Sigma=\{ \pm 1\}$ ), there is a provable near-quadratic gap in the sample complexity for realizable versus non-realizable input distributions. Note that with $O(n / \varepsilon)$ samples, we do not get accurate estimates of the mutual information edge weights. However, as we explain in Section 2, the errors for the edge weights are not independent; in fact, the errors are correlated in such a way that Chow-Liu still recovers an approximate tree! We note that our $\Omega(n / \varepsilon)$-sample complexity lower bound is specifically for recovering the structure of the unknown tree. Daskalakis, Dikkala, and Kamath [23] have shown the same lower bound for learning the distribution, but learning the tree might have been easier.

Our main tool for proving Theorem 1.2 is a result on testing conditional independence using the plug-in conditional mutual information estimator. We show that $\widetilde{O}\left(|\Sigma|^{3} / \varepsilon\right)$ samples suffice to distinguish $I(X ; Y \mid Z)=0$ from $I(X ; Y \mid Z) \geq \varepsilon$ with constant probability. In more detail:

Theorem 1.3 (Conditional Mutual Information Tester). Let $(X, Y, Z)$ be three random variables over $\Sigma$, and $(\widehat{X}, \widehat{Y}, \widehat{Z})$ be the empirical distribution over a size $N$ sample of $(X, Y, Z)$. There exists a universal constant $0<C<1$ so that for any

$$
N \geq \Theta\left(\frac{|\Sigma|^{3}}{\varepsilon} \log \frac{|\Sigma|}{\delta} \log \frac{|\Sigma| \log (1 / \delta)}{\varepsilon}\right),
$$

the following results hold with probability $1-\delta$ :

(1) If $I(X ; Y \mid Z)=0$, then $I(\widehat{X} ; \widehat{Y} \mid \widehat{Z})<\varepsilon$.

(2) If $I(X ; Y \mid Z) \geq \varepsilon$, then $I(\widehat{X} ; \widehat{Y} \mid \widehat{Z})>C \cdot I(X ; Y \mid Z)$.

We also get a similar result for unconditional mutual information testing (testing if $I(X ; Y)=0$ or $I(X ; Y) \geq \varepsilon$ ) with a $|\Sigma|$ factor smaller $N$. Conditional independence testing has previously been studied in [14], which gave optimal bounds for testing whether $(X, Y, Z)$ is conditionally independent or $\varepsilon$-far from independent in total variation distance. Developing a (conditional) independence tester with respect to mutual information with $o\left(\frac{1}{\varepsilon^{2}}\right)$ sample complexity was posed as an open problem in [14]; Theorem 1.3 resolves this with optimal $\varepsilon$ dependence. Moreover, the test statistic used by Theorem 1.3 is simply the empirical mutual information, which is key for our application to Chow-Liu.

Theorem 1.2 describes how Chow-Liu finds a good tree T. Our final result shows how to estimate the nearest $T$-structured distribution for fixed $T$. As above, the spirit of our approach is to make the algorithms as simple as possible (moving possible complications to the analysis). For the fixed-structure learning problem, the most natural approach is to empirically estimate $X_{i} \mid X_{\mathrm{pa}(i)}=x$ for each non-root node $i$ and for each setting $x$ of the parent of $i$. However, for $\mathrm{KL}$ divergence, the empirical estimator is known to not work; so, we move to the next most natural estimator: Laplace's add-1 estimator [41].

TheOREM 1.4. Let $P$ be a discrete distribution over $\Sigma^{n}$. Let $T$ be a tree on $n$ vertices, and $Q$ be a $T$-structured distribution with conditional probabilities at each node estimated using the empirical add-1 estimator on

$$
N=\Theta\left(\frac{n|\Sigma|^{2}}{\varepsilon} \log \frac{n|\Sigma|}{\delta} \log \left(\frac{n|\Sigma|}{\varepsilon} \log \frac{1}{\delta}\right)\right)
$$


samples from $P$. Then

$$
D(P \| Q)-D\left(P \| P_{T}\right) \leq \varepsilon
$$

with probability $1-\delta$.

The result actually holds for arbitrary Bayes net models, not just trees. The sample complexity becomes $\widetilde{O}\left(n|\Sigma|^{d+1} / \varepsilon\right)$ for Bayes nets with in-degree at most $d$. To the best of our knowledge, this is the first efficient algorithm with this guarantee. Combining Theorem 1.4 with Theorem 1.2 shows that, for any tree-structured distribution $P$, after $\widetilde{O}\left(|\Sigma|^{3} n / \varepsilon\right)$ samples, we can properly learn a tree-structured distribution $Q$ satisfying $D(P \| Q) \leq \varepsilon$ (and hence $\left.\|P-Q\|_{T V} \leq \sqrt{2 \varepsilon}\right)$ (see Algorithm 1).

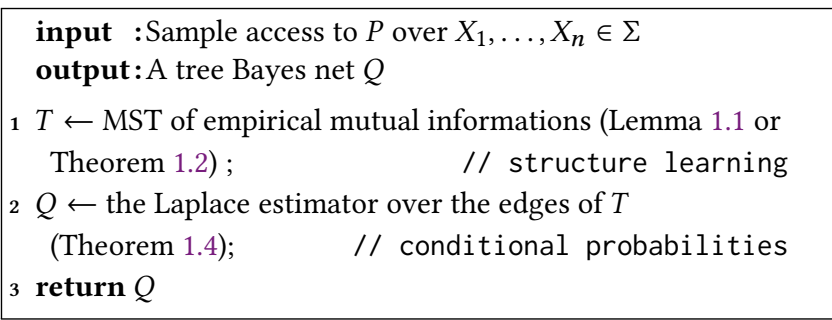

Algorithm 1: Learning Tree-structured Distributions from Samples

\subsection{Related Work}

Learning a multivariate distribution from samples is an important problem in machine learning and statistics with many applications. The problem is provably intractable for general high-dimensional multivariate distributions (e.g., [34]). Thus, structural assumptions need to be made for designing efficient and practical learning algorithms for high-dimensional distributions. Graphical models including Bayesian networks and Markov Random Fields (MRFs) are widely popular natural classes of structured distributions. In this setup, the learning problem naturally decomposes into two subproblems: structure learning and parameter learning.

For structure learning, the goal is to output the best structure (eg: a Bayes net that maximizes the likelihood of the data), given independent samples. Unfortunately, in general for both Bayes nets and MRFs, finding the best structure is known to be NPhard [17, 20, 35, 44]. In this context, Chow-Liu algorithm remains one of the few efficient structure learning algorithms that does not require any additional assumptions. Since its publication, researchers have continued to look into analyzing properties of this algorithm [18, 45, 51, 52] and generalizing it to other classes of graphs, e.g., polytrees [22], bounded treewidth graphs [47, 50], and mixtures of trees [3, 46]. Most of these works focus on establishing conditions guaranteeing that the algorithm recovers the exact tree structure in the limit that the number of samples tends to infinity. Also, for general graph-structured Ising and Markov random fields, several algorithms [7, 10, 30, 38, 57, 58] have been proposed that recover the graphical structure under various distributional assumptions.

As mentioned in the introduction, a common motivation for structure learning is to subsequently use the structure for inference algorithms. For such applications, instead of recovering the exact structure, it is more relevant to recover a model that approximates the original distribution statistically and on which inference can be performed efficiently. For example, Wainwright [55] discusses situations in which it is computationally beneficial to use inconsistent learning algorithms (even in the infinite sample limit) to feed into approximate inference algorithms. Trees play an important role for inference algorithms, since the commonly used sum-product algorithm assumes tree structure, and other more general inference algorithms (like the junction tree algorithm and various approximate inference techniques) rely on tree-like structure. This is what motivates the notion of learning $\varepsilon$-approximate trees considered in this paper.

The problem of learning $\varepsilon$-approximate graphical models has a long history. Höffgen [33] studied the $\varepsilon$-approximate structure learning of an unknown Bayes net over $\{0,1\}^{n}$ of indegree $d$ as a combinatorial optimization problem and gave a sample complexity of $\widetilde{O}\left(n^{2} 4^{d} \varepsilon^{-2}\right)^{*}$. He showed that the optimization problem is efficient for trees $(d=1)$, essentially establishing Lemma 1.1 for distributions on $\{0,1\}^{n}$. There have been several other works which provide PAC-learning guarantees for generalizations of trees: bounded treewidth junction trees [16, 47], factor graphs [1], and forest-structured MRF's [43]. While we consider the KL divergence between the true distribution and the output of Chow-Liu. Bresler and Karzand [8] recently studied the same question with respect to maximum total variation distance between pairwise marginals. Their work is restricted to Ising models, and their sample complexity depends on bounds on distributional parameters (edge weights) while ours do not. In another recent work, Brustle, Cai and Daskalakis [11] (generalizing the results in [26]) get bounds on the sample complexity of learning $\varepsilon$-approximate ${ }^{\dagger}$ MRF's with bounded hyper-edges and Bayesian networks with bounded in-degree, but they do not get efficient algorithms for these problems.

The main technical component of our analysis of the Chow-Liu algorithm is a new conditional independence tester which falls in the framework of distribution property testing $[5,32]$. We refer the reader to the surveys [12,49] and the textbook [31] and references therein for more details and results in this rapidly progressing field. Testing independence of two or more random variables has received some attention in distribution testing [2, 5, 14, 27, 29]. The simplest formulation of the problem is the following: Given samples from an unknown joint distribution on variables $(X, Y)$ : decide with probability $\geq 2 / 3$ whether $X$ and $Y$ are independent or they are $\varepsilon$ far (under some distance measure) from the product distribution $X \times Y$. Very recently, motivated by its practical importance, [14] considered the problem of conditional independence testing. In particular, given samples from an unknown discrete random variable $(X, Y, Z)$ on domain $\left[\ell_{1}\right] \times\left[\ell_{2}\right] \times[n]$, distinguish, with probability at least $2 / 3$, between the case that $X$ and $Y$ are conditionally independent given $Z$ from the case that $(X, Y, Z)$ is $\varepsilon$-far in TV distance from every distribution that has this property. The key difference in our setting is that we are interested in the stronger notion of KL divergence.

The parameter learning problem (i.e., learning the distribution with given structure) is also well-studied. Dasgupta [21] showed an

*Höffgen's capped the empirical probabilities away from 0 and 1 and then used a plug-in estimator for entropy/MI.

${ }^{\dagger}$ In total variation distance rather than KL 
$\widetilde{O}\left(n^{2} 2^{d} \log (1 / \delta) / \varepsilon^{2}\right)$ for learning an $\varepsilon$-approximate Bayes net on $n$ boolean variables with in-degree at most $d$. We improve the dependence on $n$ and $\varepsilon^{-1}$ to linear. For the realizable setting ${ }^{\dagger}$, [6] also obtained the same improvement but for constant error probability $\delta$. The key to obtaining our $\log (1 / \delta)$ dependence is a PAC analysis of the add-1 estimator, which is new to the best of our knowledge. Kamath, Orlitsky, Pichapati and Suresh [34] analyze the expected risk, which does not directly imply a high-probability bound. Recently, Bresler and Karzand [9] studied the parameter recovery problem for tree Ising models with respect to the TV distance.

Finally, we note that while our work focuses on learning tree structured distributions, recent works [6, 15, 23, 24] have investigated testing problems for more general classes of high-dimensional distributions.

Concurrent Work. During preparation of this paper, a concurrent work by Daskalakis and Pan [25] was posted online. The headline result-that Chow-Liu learns tree-structured distributions with near-linear number of samples-is the same. The techniques employed are quite different and more involved, with [25] working in squared Hellinger distance rather than KL and not involving the connection to conditional independence testing (Theorem 1.3). The details of the theorem are also somewhat different, most notably in that our result uses a $\log n$ factor more samples while [25] only works for a binary alphabet $\Sigma$.

\section{PROOF OVERVIEW}

For the purposes of this proof overview, we consider a constant size alphabet $\Sigma$.

\subsection{Finding an Approximate Tree}

For any distribution $P$ and a tree $T$, it is known that $P_{T}$ is simply the distribution that matches the marginals of $P$ on each edge of $T$. The Chow-Liu algorithm [19] is based on the following observation:

$$
D\left(P \| P_{T}\right)=J_{P}-\operatorname{wt}_{P}(T)
$$

where $J_{P}=\sum_{v} H\left(P_{v}\right)-H(P)$ is independent of $T\left(P_{v}\right.$ is the marginal on variable $v$ ), and

$$
\operatorname{wt}_{P}(T):=\sum_{(X, Y) \in T} I(X ; Y)
$$

is the weight of $T$ in the complete graph weighted by pairwise mutual information. Therefore $D\left(P \| P_{T}\right)$ is minimized when $T$ is the maximum weight spanning tree $T^{*}$ of this weighted complete graph.

The main question is how many samples are necessary for the maximum-weight spanning tree of the empirical distribution $\widehat{P}$ to have nearly-optimal weight under the true distribution $P$. That is, for Chow-Liu to recover a $\widehat{T}$ with $D\left(P \| P_{\widehat{T}}\right) \leq D\left(P \| P_{T^{*}}\right)+\varepsilon$, it is necessary and sufficient that

$$
\widehat{T}=\arg \max \mathrm{wt}_{\widehat{P}}(T)
$$

satisfies

$$
\operatorname{wt}_{P}(\widehat{T}) \geq \operatorname{wt}_{P}\left(T^{*}\right)-\varepsilon .
$$

¥ This result (for TV distance) was also claimed in the appendix of [15], but the analysis there appears to incomplete [13].
The non-realizable setting. The simplest approach to achieving (2.2) would be to ensure that $|I(\widehat{X} ; \widehat{Y})-I(X ; Y)| \leq \frac{\varepsilon}{2 n}$ for all vertex pairs $(X, Y)$. This guarantees for every $T$ that $\left|\mathrm{wt}_{\widehat{P}}(T)-\mathrm{wt}_{P}(T)\right| \leq$ $|T| \frac{\varepsilon}{2 n}<\varepsilon / 2$, which gives (2.2). Estimating mutual information to within $\frac{\varepsilon}{2 n}$ is possible with $\widetilde{\Theta}\left(n^{2} / \varepsilon^{2}\right)$ samples, with high probability. A union bound over all vertex pairs then gives the Lemma 1.1 upper bound. We also show that this bound is tight. Estimating $I(X ; Y)$ to $\pm \varepsilon$ really does require $1 / \varepsilon^{2}$ samples (for example, if $X$ is uniform on $\{0,1\}$ and $\operatorname{Pr}[Y=X]=p \approx \frac{3}{4}$, then estimating $I(X ; Y)=1-h(p)$ requires estimating $p$ to within $\pm \Theta(\varepsilon))$. We can translate this hardness into a $\Omega\left(1 / \varepsilon^{2}\right)$ lower bound for a (non-treestructured) three-variable $P$ [see Figure 1a]; and by concatenating $\Omega(n)$ of these instances together, we get an $\Omega\left(n^{2} / \varepsilon^{2}\right)$ lower bound.

The realizable setting. Fortunately, we can do much better in the realizable setting, where $P$ is actually $T^{*}$-structured for some tree $T^{*}$. We show that the errors in estimating mutual information are correlated, as illustrated in Figure 1b, so that the difference between mutual informations will be estimated more accurately than the mutual information itself.

As an example, consider the three variable case, where the true $T^{*}$ is $X-Y-Z$ and we want to ensure the algorithm does not pick edge $X Z$ over $Y Z$. We use the identity:

$$
I(Y ; Z)-I(X ; Z)=I(Y ; Z \mid X)-I(X ; Z \mid Y) \text {. }
$$

In order for picking $X Z$ over $Y Z$ to be $\varepsilon$-bad, the left hand side must be at least $\varepsilon$. On the other hand, because $P$ is $X-Y-Z$-structured, $I(X ; Z \mid Y)=0$, and hence $I(Y ; Z \mid X) \geq \varepsilon$.

Chow-Liu looks at the empirical mutual information, which has the same identity:

$$
I(\widehat{Y} ; \widehat{Z})-I(\widehat{X} ; \widehat{Z})=I(\widehat{Y} ; \widehat{Z} \mid \widehat{X})-I(\widehat{X} ; \widehat{Z} \mid \widehat{Y}) .
$$

In order for Chow-Liu to return the wrong tree by picking $\mathrm{XZ}$ over $\mathrm{YZ}$, this must be negative. For this to happen, either $I(\widehat{X} ; \widehat{Z} \mid \widehat{Y})>$ $\varepsilon / 10$ or $I(\widehat{Y} ; \widehat{Z} \mid \widehat{X}) \leq \varepsilon / 10$. This is, effectively, a question about conditional independence testing-after how many samples can we distinguish the conditionally independent distribution $(X, Z \mid Y)$ from the $\varepsilon$-far from conditionally independent distribution $(Y, Z \mid X)$ ? Our Theorem 1.3 (discussed in the next section) shows that $\widetilde{O}(1 / \varepsilon)$ samples suffice for the empirical conditional mutual information to distinguish these cases, so that (2.3) will be positive.

For the general $n$-variable case, consider the tree $\widehat{T}$ returned by Chow-Liu. We can pair up the edges in $\widehat{T} \backslash T^{*}$ with those from $T^{*} \backslash \widehat{T}$, such that each edge $W Z$ in $\widehat{T} \backslash T^{*}$ is matched to an edge $X Y \in T^{*} \backslash \widehat{T}$ along the $W \sim Z$ path in $T^{*}$. We then use the more complicated identity

$$
\begin{aligned}
& I(X ; Y)-I(W ; Z) \\
& =I(X ; Y)-I(X ; Z)+I(X ; Z)-I(W ; Z) \\
& =I(X ; Y \mid Z)-I(X ; Z \mid Y)+I(Z ; X \mid W)-I(Z ; W \mid X) .
\end{aligned}
$$

As in the three-variable case, the negative terms in this RHS are zero, and (if picking $W Z$ over $X Y$ is $\varepsilon / n$-bad) at least one of the positive terms is at least $\varepsilon /(2 n)$. If this is the case, then again Theorem 1.3 means that the empirical estimates of these terms, after $\widetilde{O}(n / \varepsilon)$ samples, will be sufficiently accurate that $I(\widehat{X} ; \widehat{Y})-I(\widehat{W} ; \widehat{Z})>0$, and hence Chow-Liu will choose $X Y$ over $W Z$. As a result, with 


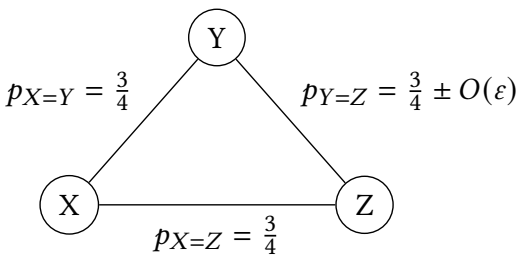

(a) Hard instance for non-realizable setting. $X, Y$, and $Z$ are individually uniform on $\{0,1\}$, and pairwise match with probability $\approx \frac{3}{4}$. Any $\varepsilon$-optimal tree will include edge $Y Z$ if $p_{Y=Z}=\frac{3}{4}+O(\varepsilon)$ and not if $p_{Y=Z}=\frac{3}{4}-O(\varepsilon)$; determining which takes $\Omega\left(1 / \varepsilon^{2}\right)$ samples.

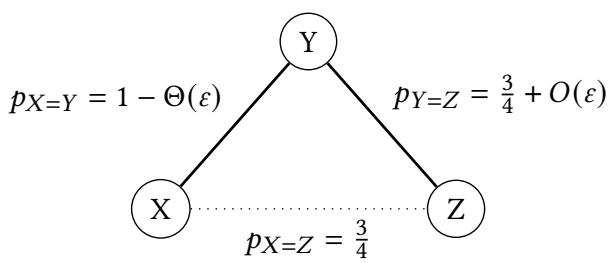

(b) A similar example in the realizable setting fails: if $P$ is actually $X-Y-Z$-structured, and $p_{Y=Z}, p_{X=Z}$ are as on the left, then $p_{X=Y}$ must be $1-O(\varepsilon)$. This means that $I(\widehat{Y} ; \widehat{Z})$ is highly correlated with $I(\widehat{X} ; \widehat{Z})$, so $I(\widehat{Y} ; \widehat{Z})-I(\widehat{X} ; \widehat{Z})$ is $\varepsilon$-accurate with only $O(1 / \varepsilon)$ samples.

\section{Figure 1: The $\Omega\left(1 / \varepsilon^{2}\right)$ bound in the non-realizable setting, and its inapplicability to the realizable setting.}

$\widetilde{O}(n / \varepsilon)$ samples, the tree $\widehat{T}$ recovered by Chow-Liu will satisfy $(2.2)$, giving Theorem 1.2.

\subsection{Conditional Independence Testing}

Independence Testing. To build up to conditional independence testing with respect to mutual information, consider unconditional independence testing: given samples $(X, Y) \sim P_{X Y}$, determine whether $I(X ; Y)$ is 0 or $\geq \varepsilon$. We would like to show that, with $O\left(\frac{1}{\varepsilon} \log \frac{1}{\varepsilon}\right)$ samples, the empirical mutual information $I(\widehat{X} ; \widehat{Y})$ will distinguish between these two cases. [Note that $\Omega\left(\frac{1}{\varepsilon} \log \frac{1}{\varepsilon}\right)$ samples are necessary even in the binary setting: if $X=Y$ always, but $\operatorname{Pr}[X=1]$ is either 0 or $\varepsilon / \log (1 / \varepsilon)$, the mutual information is either 0 or $\Theta(\varepsilon)$, but the first $\Omega\left(\frac{1}{\varepsilon} \log \frac{1}{\varepsilon}\right)$ samples will probably all be zero in either case.]

For intuition, consider the binary setting. Let $p_{y}=\operatorname{Pr}[X=1 \mid$ $Y=y]$, and $p=\operatorname{Pr}[X=1]=\mathbb{E}_{y}\left[p_{y}\right]$, so

$$
I(X ; Y)=H(X)-H(X \mid Y)=h(p)-\underset{y \sim Y}{\mathbb{E}}\left[h\left(p_{y}\right)\right]
$$

for the binary entropy function $h$. Now, estimating either $h(p)$ or the $h\left(p_{y}\right)$ to $\pm \varepsilon$ would require $1 / \varepsilon^{2}$ samples: if $p \approx \frac{1}{4}$, we would need $|\widehat{p}-p| \lesssim \varepsilon$ to estimate the individual entropies accurately. But if we expand $h\left(p_{y}\right)$ in a Taylor expansion around $p=\mathbb{E}_{y}\left[p_{y}\right]$, the constant and linear terms cancel leaving $I(X ; Y) \approx \frac{1}{2} h^{\prime \prime}(p) E_{y}\left[\left(p_{y}-\right.\right.$ $\left.p)^{2}\right]$. So distinguishing $I(X ; Y)=0$ from $I(X ; Y) \geq \varepsilon$ involves distinguishing between $\mathbb{E}\left[\left(p_{Y}-p\right)^{2}\right]=0$ and $\mathbb{E}\left[\left(p_{Y}-p\right)^{2}\right] \gtrsim \varepsilon / h^{\prime \prime}(p)$. Up to a log factor coming from $h^{\prime \prime}(p)$, at least if the distribution of $y$ is fairly balanced, this means it suffices to estimate each $p_{y}$ to within $\pm \sqrt{\varepsilon} / 10$, which takes $O(1 / \varepsilon)$ samples.

More formally and more generally, by expressing mutual information as KL and removing each entry in the sum's linear dependence on $\Delta_{x y}:=P_{x y}-P_{x} P_{y}$, we can write

$$
I(X ; Y)=D\left(P_{X Y} \| P_{X} P_{Y}\right)=\sum_{x, y \in \Sigma^{2}} f\left(\Delta_{x y}, P_{x} P_{y}\right)
$$

for some function $f$ satisfying

$$
f(a, b)=\Theta\left(\min \left(\frac{a^{2}}{b},|a| \log \left(2+\frac{|a|}{b}\right)\right)\right) .
$$

We then apply Chernoff bounds to show that every individual entry of the sum (2.4) concentrates: in the completeness case (Lemma 4.5), for any $x, y$,

$$
f\left(\widehat{\Delta}_{x y}, \widehat{P}_{x} \widehat{P}_{y}\right) \gtrsim f\left(\Delta_{x y}, P_{x} P_{y}\right)-\frac{\log N \log (1 / \delta)}{N}
$$

with probability $1-\delta$, and in the soundness case (Lemma 4.7)

$$
f\left(\widehat{\Delta}_{x y}, \widehat{P}_{x} \widehat{P}_{y}\right) \lesssim \frac{\log N \log (1 / \delta)}{N} .
$$

Taking a union bound over $X$ and $Y$, and plugging this into the sum (2.4), gives the desired tester: as long as $\frac{N}{\log N} \gtrsim \Sigma^{2} \frac{\log (\Sigma / \delta)}{\varepsilon}$, the empirical mutual information will distinguish between $I(X ; Y)>\varepsilon$ and $I(X ; Y)=0$.

The proofs of Lemma 4.5 and Lemma 4.7 are somewhat technical, but straightforward. We give intuition for the soundness case and constant probability. We use the two branches of $f$ depending on whether $P_{x y}=P_{x} P_{y}$ is large or small. If $P_{x y} \lesssim 1 / N$, then we will typically have $\widehat{\Delta}_{x y} \leq \widehat{P}_{x y} \lesssim 1 / N$, so

$$
f\left(\widehat{\triangle}_{x y}, \widehat{P}_{x} \widehat{P}_{y}\right) \lesssim \widehat{\Delta}_{x y} \log N \lesssim \frac{\log N}{N} .
$$

On the other hand, if $P_{x y} \gg 1 / N$, then typically $\widehat{P}_{x}=\Theta\left(P_{x}\right)$, $\widehat{P}_{y}=\Theta\left(P_{y}\right)$, and (we show) $\left|\widehat{\triangle}_{x y}\right| \lesssim \sqrt{P_{x} P_{y} / N}$. Hence

$$
f\left(\widehat{\Delta}_{x y}, \widehat{P}_{x} \widehat{P}_{y}\right) \lesssim \frac{\widehat{\Delta}_{x y}^{2}}{\widehat{P}_{x} \widehat{P}_{y}} \lesssim \frac{1}{N} .
$$

Conditional independence testing. By definition,

$$
I(X ; Y \mid Z)=\sum_{z} \operatorname{Pr}[Z=z] I(X ; Y \mid Z=z) .
$$

Given $N$ samples of $(X, Y, Z)$, we expect about $N \operatorname{Pr}[Z=z]$ samples from $(X ; Y \mid Z=z)$. This means our unconditional mutual independence tester will distinguish $I(X ; Y \mid Z=z)=0$ from $I(X ; Y \mid Z=z)>\frac{|\Sigma|^{2}}{\widetilde{\Omega}(N \operatorname{Pr}[Z=z])}$. If the distribution passes all these independence checks, then

$$
I(X ; Y \mid Z) \leq \sum_{z} \operatorname{Pr}[Z=z] \frac{|\Sigma|^{2}}{\widetilde{\Omega}(N \operatorname{Pr}[Z=z])}=\frac{|\Sigma|^{3}}{\widetilde{\Omega}(N)} .
$$

Thus $N=\widetilde{O}\left(|\Sigma|^{3} / \varepsilon\right)$ samples suffice to test conditional independence. A bit more care shows that the empirical conditional mutual information works as a test statistic, achieving Theorem 1.3. 


\subsection{Distribution Learning with Known Structure}

For Theorem 1.4, it is implicit in $[19,21]$ that it suffices to learn the conditional distributions in KL. While the empirical add- 1 estimator of a discrete distribution was known to have small expected $\mathrm{KL}$ error with $\widetilde{O}(|\Sigma| / \varepsilon)$ samples [34], to our knowledge a high-probability bound was not known. We use a similar analysis to our independence tester-including the same decomposition (2.4) of KL-to show that the empirical add-1 estimator is accurate with high probability (Theorem 6.1). We then show that our samples from $P$ give enough samples from each individual conditional distribution to estimate $P$ well.

\subsection{Questions}

A natural question is whether the $|\Sigma|^{3}$ dependence in our bounds can be improved. The $|\Sigma|^{3}$ term is necessary to achieve Theorem 1.3 as stated; with fewer measurements, in the soundness case of a perfectly uniform distribution, the empirical conditional mutual information will exceed $\varepsilon$. However, it is quite possible that the empirical conditional mutual information-though $\gg \varepsilon$-is still smaller than in the completeness case. Just such behavior occurs when using the empirical total variation statistic in testing total variation from uniformity [28].

Another natural question is whether one can reduce tree structure learning to conditional independence testing as a black box. The Chow-Liu algorithm only considers pairwise mutual information, and never looks at conditional mutual information at all. Our analysis introduces conditional mutual information through (2.3), which relies on the test statistic being the empirical mutual information. If future work develops better conditional independence testers based on different test statistics, does that imply more sample-efficient (but possibly slower) algorithms for tree structure learning?

Recovering the structure of an unknown bounded degree Bayesian network remains an outstanding open question. Recently, Brustle, Cai, and Daskalakis [11] have settled the sample complexity of this problem. But finding a polynomial time algorithm remains a challenge, even if we assume a correct topological ordering of the variables.

\subsection{Organization}

The rest of the paper is organized as follows. Section 3 describes the background and fixes notation. Section 4 analyzes conditional independence testing via empirical mutual information (Theorem 1.3). Section 5 uses this to show that Chow-Liu recovers an $\varepsilon$-approximate tree (Theorem 1.2). Section 6 shows how to recover the distribution given the tree (Theorem 1.4). Finally, Section 7 gives lower bounds for finding an $\varepsilon$-approximate tree $T$, showing that Lemma 1.1 and Theorem 1.2 are nearly optimal.

\section{NOTATION AND PRELIMINARIES}

For an undirected tree $T$, a rooted orientation of $T$ fixes a root vertex and orients the edges outwards from it. For a rooted orientation of $T$, if $i$ is a vertex in $T$, pa( $i$ ) denotes its parent node if any, and $\operatorname{nd}(i)$ denotes the subset of vertices not reachable from $i$.
Definition 3.1 (Tree-structured distributions). Let $T$ be a tree. Fix any rooted orientation of $i t$. Label the nodes of $T$ in topological order (so, node 1 is the root). A probability distribution $P$ over $X=\left(X_{1}, \ldots, X_{n}\right) \in \Sigma^{n}$ is said to be $T$-structured if: every variable $X_{i}$ is conditionally independent of $\left\{X_{j}: j \in \operatorname{nd}(i)\right\}$ given $X_{\mathrm{pa}(i)}$.Equivalently, $P$ admits the following factorization:

$$
\operatorname{Pr}[X=x]:=\operatorname{Pr}\left[X_{1}=x_{1}\right] \cdot \prod_{i=2}^{n} \operatorname{Pr}\left[X_{i}=x_{i} \mid X_{\mathrm{pa}(i)}=x_{\mathrm{pa}(i)}\right]
$$

A tree-structured distribution is T-structured for some tree $T$.

The following classical result justifies why the rooted orientation does not matter in Definition 3.1.

Theorem 3.2 ([54]). Let $T$ be a tree on $n$ variables, and suppose $P$ is a T-structured distribution on $\left(X_{1}, \ldots, X_{n}\right)$. For any 3 nodes $i, j, k \in[n]$, if the unique path between $i$ and $k$ in $T$ passes through $j$, then $X_{i}$ and $X_{k}$ are independent conditioned on $X_{j}$.

To compare distributions, we use the well-known notion of KLdivergence. Given two discrete probability distributions $P$ and $Q$ over $\Sigma$, their KL-divergence is defined as ${ }^{\S}$

$$
D(P \| Q):=\sum_{x \in \Sigma} P(x) \log \frac{P(x)}{Q(x)} .
$$

Recall that we say a tree $T$ is $\varepsilon$-approximate for a distribution $P$ if there exists a $T$-structured distribution $Q_{T}$ such that:

$$
D\left(P \| Q_{T}\right) \leq \varepsilon+\min _{\text {tree } T^{\prime}} \min _{\begin{array}{c}
T^{\prime} \text {-structured } \\
\text { distribution } Q^{\prime}
\end{array}} D\left(P \| Q^{\prime}\right) .
$$

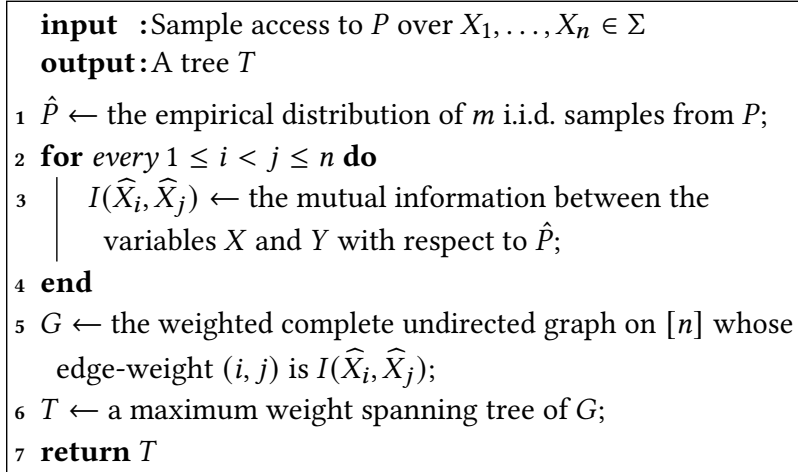

Algorithm 2: Learning the Skeleton of Tree-structured Distributions from Samples

The following lemma is implicit in [19,21].

Lemma 3.3. For a fixed tree $T$, let pa(v) denote the parent of $v$ in $T$ (or $\perp$ ifv is the root). Let $X \sim P$ and $X^{\prime} \sim Q$ for some $T$-structured $Q$. Then, if $D(P \| Q)$ is bounded:

$$
\begin{aligned}
& D(P \| Q)=\left(-H(X)+\sum_{v \in V} H\left(X_{v}\right)\right)-\sum_{v \in V} I\left(X_{v} ; X_{p a(v)}\right) \\
& +\sum_{v \in V} \sum_{x \in \Sigma} \operatorname{Pr}\left[X_{p a(v)}=x\right] D\left(X_{v}\left|X_{p a(v)}=x \| X_{v}^{\prime}\right| X_{p a(v)}^{\prime}=x\right)
\end{aligned}
$$

$\S$ All logarithms in this paper are natural, so we measure information-theoretic quantities in nats not bits. 
Lemma 3.3 is a generalization of (2.1): since $\mathrm{KL}$ divergence is nonnegative, the $Q=P_{T}$ minimizing $D(P \| Q)$ has every entry in the final sum is zero, which happens if $Q$ matches the marginals of $P$ on each edge of $T$. In that case, $D\left(P \| P_{T}\right)$ drops the final sum and gives (2.1), which we write formally:

Corollary 3.4 ([19]). Let $P$ be a distribution over $\Sigma^{n}$ and $T$ be an undirected tree over the vertex set $[n]$. Let $P_{T}$ be the most likely distribution of $P$ for the tree $T$. Then,

$$
D\left(P \| P_{T}\right)=J_{P}-\mathrm{wt}_{P}(T)
$$

where $J_{P}=\sum_{v} H\left(P_{v}\right)-H(P)$ is independent of $T$ ( $P_{v}$ is the marginal on variable $v)$, and $\operatorname{wt}_{P}(T):=\sum_{(X, Y) \in T} I(X ; Y)$.

This suggests the Chow-Liu algorithm (see Algorithm 2) that we analyze.

\section{TESTING INDEPENDENCE AND CONDITIONAL INDEPENDENCE}

Setup. We assume all random variables are over a discrete domain $\Sigma$. Let $X$ and $Y$ be random variables over $\Sigma$ distributed jointly according to $P$. For any pair $x, y \in \Sigma$, let $P_{x}, P_{y}, P_{x y}$ denote $\operatorname{Pr}[X=$ $x], \operatorname{Pr}[Y=y], \operatorname{Pr}[(X, Y)=(x, y)]$, respectively. Let $\Delta_{x y}:=P_{x y}-$ $P_{x} P_{y}$. Hence $\sum_{x y} \Delta_{x y}=0$. Let $(\widehat{X}, \widehat{Y})$ be the random variable distributed according to the empirical distribution $\widehat{P}$ over $(X, Y)$ over a finite set of independent samples. Let $\widehat{P}_{x}, \widehat{P}_{y}, \widehat{P}_{x y}, \widehat{\Delta}_{x y}$ denote the same values for $(\widehat{X}, \widehat{Y})$.

Define

$$
f(a, b):=(a+b) \log (1+a / b)-a
$$

for all $b \in[0,1], a \in[-b, 1-b]$ [with $f(-b, b)=b$, being the limiting value].

Claim 4.1. For two random variables $X$ and $Y$ over $\Sigma, I(X ; Y)=$ $\sum_{x, y} f\left(\Delta_{x y}, P_{x} P_{y}\right)$.

Proof.

$$
\begin{aligned}
I(X ; Y) & =D\left(P_{X Y} \| P_{X} P_{Y}\right) \\
& =\sum_{x, y}\left(P_{x} P_{y}+\Delta_{x y}\right) \log \left(1+\Delta_{x y} /\left(P_{x} P_{y}\right)\right) \\
& =\sum_{x, y}\left[\left(P_{x} P_{y}+\Delta_{x y}\right) \log \left(1+\Delta_{x y} /\left(P_{x} P_{y}\right)\right)-\Delta_{x y}\right] \\
& =\sum_{x, y} f\left(\Delta_{x y}, P_{x} P_{y}\right) .
\end{aligned}
$$

\subsection{Analysis of $f$}

LemmA 4.2. For any $a \geq-b$ and $b \geq 0$,

$$
f(a, b)=C_{a, b} \min \left(\frac{a^{2}}{b},|a| \log \left(2+\frac{|a|}{b}\right)\right)
$$

where the coefficient $1 / 3 \leq C_{a, b} \leq 1$.

Proof. Using Calculus and Taylor expansion.

Claim 4.3. For any $x, y$, the following holds:

(1) $f\left(\Delta_{x y}, P_{x} P_{y}\right) \leq 1$.

(2) $\min \left(P_{x}, P_{y},\left|\Delta_{x y}\right|\right) \gtrsim f\left(\Delta_{x y}, P_{x} P_{y}\right) / \log \left(3 / f\left(\Delta_{x y}, P_{x} P_{y}\right)\right)$.
Proof. Of (1): WLOG $P_{x} \leq P_{y}$. Note that $-P_{x} P_{y} \leq \Delta_{x y} \leq$ $P_{x}-P_{x} P_{y}$, and $f(x, a)$ is convex in $x$, so that:

$$
\begin{aligned}
f\left(\triangle_{x y}, P_{x} P_{y}\right) & \leq \max \left(f\left(-P_{x} P_{y}, P_{x} P_{y}\right), f\left(P_{x}-P_{x} P_{y}, P_{x} P_{y}\right)\right) \\
& \leq \max \left(P_{x} P_{y}, P_{x} \log \left(1+1 / P_{y}\right)-P_{x}+P_{x} P_{y}\right) \\
& \leq \max \left(1, P_{x} \log \left(1+1 / P_{x}\right)\right) \\
& \leq 1
\end{aligned}
$$

Of (2): We have

$$
\begin{aligned}
& f\left(\Delta_{x y}, P_{x} P_{y}\right) \\
& \lesssim\left|\Delta_{x y}\right| \log \left(2+\frac{\left|\Delta_{x y}\right|}{P_{x} P_{y}}\right) \leq P_{x} \log \left(2+\frac{1}{P_{y}}\right) \leq P_{x} \log \left(\frac{3}{P_{x}}\right)
\end{aligned}
$$

and hence

$$
\min \left(P_{x}, P_{y}\right) \gtrsim f\left(\Delta_{x y}, P_{x} P_{y}\right) / \log \left(3 / f\left(\Delta_{x y}, P_{x} P_{y}\right)\right) .
$$

But then

$$
\begin{aligned}
\left|\Delta_{x y}\right| & \gtrsim f\left(\Delta_{x y}, P_{x} P_{y}\right) / \log \left(\frac{3}{P_{y}}\right) \\
& \gtrsim f\left(\Delta_{x y}, P_{x} P_{y}\right) / \log \left(3 / f\left(\Delta_{x y}, P_{x} P_{y}\right)\right),
\end{aligned}
$$

finishing the result.

\subsection{Properties of the Empirical Distribution}

By Chernoff bounds, the empirical distribution is close to the actual one:

Claim 4.4. Let $\widehat{P}_{x}, \widehat{P}_{y}$, and $\widehat{P}_{x y}$ be empirical distributions over $N>1$ samples. Then with $1-3 \delta$ probability, all of the following bounds hold:

$$
\begin{aligned}
\left|\widehat{P}_{x}-P_{x}\right| & \lesssim \sqrt{\frac{P_{x} \log \frac{2}{\delta}}{N}}+\frac{\log \frac{2}{\delta}}{N} \\
\left|\widehat{P}_{y}-P_{y}\right| & \lesssim \sqrt{\frac{P_{x} \log \frac{2}{\delta}}{N}}+\frac{\log \frac{2}{\delta}}{N} \\
\left|\widehat{P}_{x y}-P_{x y}\right| & \lesssim \sqrt{\frac{P_{x y} \log \frac{2}{\delta}}{N}}+\frac{\log \frac{2}{\delta}}{N} \\
\left|\widehat{P}_{x} \widehat{P}_{y}-P_{x} P_{y}\right| & \lesssim \sqrt{P_{x} P_{y} \frac{\log \frac{2}{\delta}}{N}}+\left(P_{x}+P_{y}\right) \frac{\log \frac{2}{\delta}}{N}+\frac{\log ^{2} \frac{2}{\delta}}{N^{2}} \\
\left|\widehat{\Delta}_{x y}-\Delta_{x y}\right| & \lesssim \sqrt{\left|\Delta_{x y}\right| \frac{\log \frac{2}{\delta}}{N}}+\sqrt{P_{x} P_{y} \frac{\log \frac{2}{\delta}}{N}}+\frac{\log \frac{2}{\delta}}{N}+\frac{\log ^{2} \frac{2}{\delta}}{N^{2}} .
\end{aligned}
$$

Proof. By the multiplicative Chernoff bound,

$$
\operatorname{Pr}\left[\left|\widehat{P}_{x}-P_{x}\right|>\varepsilon P_{x}\right]<2 \exp \left(-C \min \left(\varepsilon, \varepsilon^{2}\right) P_{x} N\right) .
$$

Rearranging, with probability $1-\delta$,

$$
\left|\widehat{P}_{x}-P_{x}\right| \lesssim \max \left(\frac{\log \frac{2}{\delta}}{P_{x} N}, \sqrt{\frac{\log \frac{2}{\delta}}{P_{x} N}}\right) P_{x} \leq \sqrt{\frac{P_{x} \log \frac{2}{\delta}}{N}}+\frac{\log \frac{2}{\delta}}{N} .
$$

Similarly for $\widehat{P}_{y}$ and $\widehat{P}_{x y}$. Then

$$
\begin{aligned}
& \left|\widehat{P}_{x} \widehat{P}_{y}-P_{x} P_{y}\right| \\
& \lesssim \sqrt{\frac{\log \frac{2}{\delta}}{N}}\left(P_{x} \sqrt{P_{y}}+P_{y} \sqrt{P_{x}}\right)+\sqrt{P_{x} P_{y}} \frac{\log \frac{2}{\delta}}{N}+\left(P_{x}+P_{y}\right) \frac{\log \frac{2}{\delta}}{N}+
\end{aligned}
$$




$$
\begin{gathered}
\left(\sqrt{P_{x}}+\sqrt{P_{y}}\right) \frac{\log ^{1.5} \frac{2}{\delta}}{N^{1.5}}+\frac{\log ^{2} \frac{2}{\delta}}{N^{2}} \\
\leq 2 \sqrt{P_{x} P_{y} \frac{\log \frac{2}{\delta}}{N}}+2\left(P_{x}+P_{y}\right) \frac{\log \frac{2}{\delta}}{N}+2\left(\sqrt{P_{x}+P_{y}}\right) \frac{\log 1.5 \frac{2}{\delta}}{N^{1.5}}+ \\
\frac{\log ^{2} \frac{2}{\delta}}{N^{2}} \\
\leq 2 \sqrt{P_{x} P_{y} \frac{\log \frac{2}{\delta}}{N}}+3\left(P_{x}+P_{y}\right) \frac{\log \frac{2}{\delta}}{N}+2 \frac{\log ^{2} \frac{2}{\delta}}{N^{2}} .
\end{gathered}
$$

This implies:

$$
\begin{aligned}
\left|\widehat{\Delta}_{x y}-\Delta_{x y}\right| & \leq\left|\widehat{P}_{x y}-P_{x y}\right|+\left|\widehat{P}_{x} \widehat{P}_{y}-P_{x} P_{y}\right| \\
& \lesssim \sqrt{P_{x y} \frac{\log \frac{2}{\delta}}{N}}+\sqrt{P_{x} P_{y} \frac{\log \frac{2}{\delta}}{N}}+\frac{\log \frac{2}{\delta}}{N}+\frac{\log ^{2} \frac{2}{\delta}}{N^{2}} .
\end{aligned}
$$

The result follows because $P_{x y} \leq 2 \Delta_{x y}$ whenever that term is largest.

\subsection{Completeness}

LEMMA 4.5. Let $\widehat{P}$ be the empirical distribution over $N$ samples. Then there exist constants $C, C^{\prime}>0$ such that for every $\delta>0$ if $f\left(\Delta_{x y}, P_{x} P_{y}\right) \geq C \frac{\log N}{N} \log \frac{2}{\delta}$, then:

$$
\operatorname{Pr}\left[f\left(\widehat{\Delta}_{x y}, \widehat{P}_{x} \widehat{P}_{y}\right)>C^{\prime} f\left(\Delta_{x y}, P_{x} P_{y}\right)\right]>1-3 \delta .
$$

Proof. By Claim $4.3 f\left(\Delta_{x y}, P_{x} P_{y}\right) \leq 1$. Claim 4.3 also implies

$$
\min \left(\left|\Delta_{x y}\right|, P_{x}, P_{y}\right) \gtrsim \frac{f\left(\Delta_{x y}, P_{x} P_{y}\right)}{\log \left(2 / f\left(\Delta_{x y}, P_{x} P_{y}\right)\right)} \gtrsim \frac{C}{N} \log \frac{2}{\delta} .
$$

Suppose that the Claim 4.4 statements hold, as happens with $1-3 \delta$ probability. We will show that this implies the result.

We split into cases, based on whether $\Delta_{x y}>8 P_{x} P_{y}$.

Large $\Delta_{x y}$. This case of $\Delta_{x y}>8 P_{x} P_{y}$ implies

$$
f\left(\Delta_{x y}, P_{x} P_{y}\right) \approx \Delta_{x y} \log \frac{\Delta_{x y}}{P_{x} P_{y}} .
$$

In this regime, we have by Claim 4.4 holding that

$$
\left|\widehat{\Delta}_{x y}-\Delta_{x y}\right| \lesssim \sqrt{\frac{\Delta_{x y}}{N} \log \frac{2}{\delta}}+\frac{\log \frac{2}{\delta}}{N} .
$$

Since $N \gtrsim C \log \frac{2}{\delta} / \Delta_{x y}$ by (4.2), this implies

$$
\left|\widehat{\Delta}_{x y}-\Delta_{x y}\right| \lesssim \Delta_{x y} / C
$$

and hence $\left|\widehat{\Delta}_{x y}-\Delta_{x y}\right|<\Delta_{x y} / 10$ for a sufficiently large $C$.

We also have by Claim 4.4 holding that

$$
\begin{aligned}
\left|\widehat{P}_{x} \widehat{P}_{y}-P_{x} P_{y}\right| & \lesssim \sqrt{P_{x} P_{y} \frac{\log \frac{2}{\delta}}{N}}+\left(P_{x}+P_{y}\right) \frac{\log \frac{2}{\delta}}{N}+\frac{\log ^{2} \frac{2}{\delta}}{N^{2}} \\
& \lesssim \sqrt{\frac{\Delta_{x y}}{N} \log \frac{2}{\delta}}+\frac{\log \frac{2}{\delta}}{N}
\end{aligned}
$$

and hence (by (4.2)) $\left|\widehat{P}_{x} \widehat{P}_{y}-P_{x} P_{y}\right| \leq \Delta_{x y} / 10$ for a sufficiently large C. This implies $\widehat{P}_{x} \widehat{P}_{y} \leq 0.23 \Delta_{x y}$. Therefore:

$$
\frac{\widehat{\Delta}_{x y}}{\widehat{P}_{x} \widehat{P}_{y}} \geq \frac{0.9 \Delta_{x y}}{0.23 \Delta_{x y}}>3.9
$$

so that (in Lemma 4.2),

$$
f\left(\widehat{\Delta}_{x y}, \widehat{P}_{x} \widehat{P}_{y}\right) \approx \widehat{\Delta}_{x y} \log \frac{\widehat{\Delta}_{x y}}{\widehat{P}_{x} \widehat{P}_{y}} \gtrsim \Delta_{x y} \log \frac{\Delta_{x y}}{\widehat{P}_{x} \widehat{P}_{y}}
$$

Now,

$$
\begin{aligned}
\left|\widehat{P}_{x} \widehat{P}_{y}-P_{x} P_{y}\right| & \lesssim \sqrt{P_{x} P_{y} \frac{\log \frac{2}{\delta}}{N}}+\left(P_{x}+P_{y}\right) \frac{\log \frac{2}{\delta}}{N}+\frac{\log ^{2} \frac{2}{\delta}}{N^{2}} \\
& \lesssim \sqrt{P_{x} P_{y} \Delta_{x y} / C}+2 P_{x} P_{y} / C+P_{x} P_{y} / C^{2} \\
& \lesssim \frac{1}{\sqrt{C}}\left(P_{x} P_{y}+\sqrt{P_{x} P_{y} \Delta_{x y}}\right) .
\end{aligned}
$$

For sufficiently large constant $C$ the constant factor is overcome, so that

$$
\begin{aligned}
f\left(\widehat{\Delta}_{x y}, \widehat{P}_{x} \widehat{P}_{y}\right) & \approx \Delta_{x y} \log \frac{\Delta_{x y}}{2 P_{x} P_{y}+\sqrt{P_{x} P_{y} \Delta_{x y}}} \\
& \geq \Delta_{x y} \min \left(\log \frac{\Delta_{x y}}{4 P_{x} P_{y}}, \log \frac{\Delta_{x y}}{2 \sqrt{P_{x} P_{y} \Delta_{x y}}}\right) \\
& \approx \Delta_{x y} \min \left(\log \frac{\Delta_{x y}}{P_{x} P_{y}}, \frac{1}{2} \log \frac{\Delta_{x y}}{P_{x} P_{y}}\right) \\
& \approx f\left(\Delta_{x y}, P_{x} P_{y}\right)
\end{aligned}
$$

as desired.

Small $\Delta_{x y}$. This case of $-P_{x} P_{y} \leq \Delta_{x y} \leq 8 P_{x} P_{y}$ implies

$$
f\left(\Delta_{x y}, P_{x} P_{y}\right) \approx \Delta_{x y}^{2} /\left(P_{x} P_{y}\right) \leq 64 P_{x} P_{y} .
$$

Now, the condition that $f\left(\Delta_{x y}, P_{x} P_{y}\right)>C \frac{\log N}{N} \log \frac{2}{\delta}$ implies

$$
P_{x} P_{y} \geq \frac{1}{64} \Delta_{x y}^{2} /\left(P_{x} P_{y}\right) \gtrsim C \frac{\log N}{N} \log \frac{2}{\delta}
$$

and hence $N \gtrsim C \log \frac{2}{\delta} / \min \left(P_{x}, P_{y}\right)$. Therefore, for a sufficiently large $C$, we have by Claim 4.4 that both:

$$
\begin{aligned}
& \left|\widehat{P}_{x}-P_{x}\right| \leq P_{x} / 10 \\
& \left|\widehat{P}_{y}-P_{x}\right| \leq P_{y} / 10
\end{aligned}
$$

Furthermore, the condition (4.3) also implies:

$$
\left|\Delta_{x y}\right| \gtrsim \sqrt{\frac{C P_{x} P_{y}}{N} \log \frac{2}{\delta}} .
$$

Hence by Claim 4.4 and the conditions (4.3) and (4.4),

$\left|\widehat{\Delta}_{x y}-\Delta_{x y}\right| \lesssim \sqrt{\frac{P_{x} P_{y}}{N} \log \frac{2}{\delta}}+\frac{\log \frac{2}{\delta}}{N} \lesssim\left|\Delta_{x y}\right| / \sqrt{C}+\Delta_{x y}^{2} /\left(P_{x} P_{y} C\right)$.

Using $\left|\Delta_{x y}\right| \leq 8 P_{x} P_{y}$, we get $\left|\widehat{\Delta}_{x y}-\Delta_{x y}\right|<\left|\Delta_{x y}\right| / 10$ for a sufficiently large constant $C$. Therefore:

$$
f\left(\widehat{\Delta}_{x y}, \widehat{P}_{x} \widehat{P}_{y}\right) \gtrsim \frac{\widehat{\Delta}_{x y}^{2}}{\widehat{P}_{x} \widehat{P}_{y}} \approx \frac{\Delta_{x y}^{2}}{P_{x} P_{y}} \approx f\left(\Delta_{x y}, P_{x} P_{y}\right) .
$$

COROLlary 4.6. Let $\widehat{P}$ be the empirical distribution over $N>1$ samples. Then there exist universal constants $C_{1}, C_{2}>0$ such that for every $\delta>0$ :

$$
I(\widehat{X} ; \widehat{Y}) \geq C_{1} I(X ; Y)-C_{2}|\Sigma|^{2} \frac{\log N}{N} \log \frac{|\Sigma|}{\delta}
$$


with probability at least $1-\delta$.

Proof. Lemma 4.5 has a condition on $f$ being large. But in general, since $f \geq 0$ always, it shows that with probability $1-3 \delta$,

$$
f\left(\widehat{\Delta}_{x y}, \widehat{P}_{x} \widehat{P}_{y}\right)>C^{\prime} f\left(\Delta_{x y}, P_{x} P_{y}\right)-C^{\prime} C \frac{\log N}{N} \log \frac{2}{\delta} .
$$

Taking a union bound over the sum (4.1), and rescaling $\delta$ by $3|\Sigma|^{2}$, we get the result.

\subsection{Soundness}

LEMMA 4.7. Let $\widehat{P}$ be the empirical distribution over $N$ samples. Then there exists a universal constant $C>0$ such that for every $\delta>0$, if $\Delta_{x y}=0$ then:

$$
\operatorname{Pr}\left[f\left(\widehat{\Delta}_{x y}, \widehat{P}_{x} \widehat{P}_{y}\right)<C \frac{\log N}{N} \log \frac{2}{\delta}\right]>1-3 \delta .
$$

Proof. As in the completeness section, we suppose that the equations of Claim 4.4 all hold. In particular, this implies that

$$
\left|\widehat{\Delta}_{x y}\right| \lesssim \sqrt{\frac{P_{x} P_{y}}{N} \log \frac{2}{\delta}}+\frac{\log \frac{2}{\delta}}{N} .
$$

We again split into cases depending on $P_{x} P_{y}<\frac{\log \frac{2}{\delta}}{C N}$ or not for a large enough constant $C$.

$$
\text { Small } \begin{aligned}
P_{x} P_{y} \text {. Suppose } P_{x} P_{y} & <\frac{\log \frac{2}{\delta}}{C N} \text {, so that } \\
\left|\widehat{\Delta}_{x y}\right| & \lesssim \frac{1}{N} \log \frac{2}{\delta} .
\end{aligned}
$$

First note that, if either of $\widehat{P}_{x}=0$ or $\widehat{P}_{y}=0$, then $\widehat{P}_{x y}=0$ and $\widehat{\Delta}_{x y}=0$, so $f\left(\widehat{\Delta}_{x y}, \widehat{P}_{x} \widehat{P}_{y}\right)=0$. Therefore, in order for $f\left(\widehat{\Delta}_{x y}, \widehat{P}_{x} \widehat{P}_{y}\right)$ to be nonzero, we must sample $x$ and $y$ in our set, in which case $\widehat{P}_{x} \widehat{P}_{y} \geq 1 / N^{2}$.

Therefore

$$
\begin{aligned}
f\left(\widehat{\Delta}_{x y}, \widehat{P}_{x} \widehat{P}_{y}\right) & \lesssim\left|\widehat{\Delta}_{x y}\right| \log \left(1+\widehat{\Delta}_{x y} /\left(\widehat{P}_{x} \widehat{P}_{y}\right)\right) \\
& \lesssim\left|\widehat{\Delta}_{x y}\right| \log \left(1+N^{2}\right) \lesssim \frac{1}{N} \log \frac{2}{\delta} \log N
\end{aligned}
$$

Large $P_{x} P_{y}$. If $P_{x} P_{y} \geq \frac{\log \frac{2}{\delta}}{C N}$, then

$$
\min \left(P_{x}, P_{y}\right) \geq \frac{\log \frac{2}{\delta}}{C N}
$$

and hence by Claim 4.4 holding we have

$$
\left|\widehat{P}_{x}-P_{x}\right| \leq O\left(\sqrt{\frac{P_{x}}{N} \log \frac{2}{\delta}}+\frac{\log \frac{2}{\delta}}{N}\right) \leq P_{x} / 2,
$$

for a large enough $C$ and similarly for $\widehat{P_{y}}$. Therefore:

$f\left(\widehat{\Delta}_{x y}, \widehat{P}_{x} \widehat{P}_{y}\right) \lesssim \frac{\widehat{\Delta}_{x y}^{2}}{\widehat{P}_{x} \widehat{P}_{y}} \leq 4 \frac{\widehat{\Delta}_{x y}^{2}}{P_{x} P_{y}} \lesssim \frac{1}{N} \log \frac{2}{\delta}+\frac{\log ^{2} \frac{2}{\delta}}{N^{2} P_{x} P_{y}} \lesssim \frac{C}{N} \log \frac{2}{\delta}$.

Therefore the result holds regardless of the case, as long as Claim 4.4 holds.

COROLlary 4.8. Let $\widehat{P}$ be the empirical distribution over $N>1$ samples. There exists a universal constant $C_{3}>0$ such that if $P$ is a product distribution, then for every $\delta>0$ :

$$
I(\widehat{X} ; \widehat{Y}) \leq \frac{\log N}{N} C_{3}|\Sigma|^{2} \log \frac{|\Sigma|}{\delta}
$$

with probability at least $1-\delta$.

Proof. Follows from taking the sum (4.1) and applying a union bound over the events in Lemma 4.7 for all possible $x, y$.

\subsection{Conditional Independence Testing}

Theorem 1.3 (Conditional Mutual Information Tester). Let $(X, Y, Z)$ be three random variables over $\Sigma$, and $(\widehat{X}, \widehat{Y}, \widehat{Z})$ be the empirical distribution over a size $N$ sample of $(X, Y, Z)$. There exists a universal constant $0<C<1$ so that for any

$$
N \geq \Theta\left(\frac{|\Sigma|^{3}}{\varepsilon} \log \frac{|\Sigma|}{\delta} \log \frac{|\Sigma| \log (1 / \delta)}{\varepsilon}\right),
$$

the following results hold with probability $1-\delta$ :

(1) If $I(X ; Y \mid Z)=0$, then $I(\widehat{X} ; \widehat{Y} \mid \widehat{Z})<\varepsilon$.

(2) If $I(X ; Y \mid Z) \geq \varepsilon$, then $I(\widehat{X} ; \widehat{Y} \mid \widehat{Z})>C \cdot I(X ; Y \mid Z)$.

Proof. For any $z \in \Sigma$ let $N_{z}$ be the number of samples with $Z=z$.

Proof of (1): If $I(X ; Y \mid Z)=0$ then $I(X ; Y \mid Z=z)=0$ for each $z$. Then Corollary 4.8 gives us that, with probability at least $1-\delta$,

$$
I(\widehat{X} ; \widehat{Y} \mid \widehat{Z}=z) \lesssim \frac{|\Sigma|^{2}}{N_{z}} \log \frac{|\Sigma|}{\delta} \log N_{z} \leq \frac{|\Sigma|^{2}}{N_{z}} \log \frac{|\Sigma|}{\delta} \log N .
$$

Let $S \subseteq \Sigma$ contain the set of $z$ such that $\operatorname{Pr}\left[\left|\widehat{P}_{z}-P_{z}\right|>P_{z} / 2\right] \leq \delta$. By a Chernoff bound, this consists of all $z$ with $P_{z} \geq O\left(\frac{\log 1 / \delta}{N}\right)$. With probability $1-2|\Sigma| \delta$, then,

$$
\begin{aligned}
\sum_{z \in S} \widehat{P}_{z} I(\widehat{X} ; \widehat{Y} \mid \widehat{Z}=z) & \lesssim \sum_{z \in S} P_{z} \frac{|\Sigma|^{2}}{N P_{z} / 2} \log \frac{|\Sigma|}{\delta} \log N \\
& \leq \frac{\log N}{N} 2|\Sigma|^{3} \log \frac{|\Sigma|}{\delta} .
\end{aligned}
$$

On the other hand, $z \notin S$ will have $\widehat{P}_{z} \lesssim \frac{\log (1 / \delta)}{N}$ with probability $1-\delta$, so that with probability $1-|\Sigma| \delta$

$$
\sum_{z \notin S} \widehat{P}_{z} I(\widehat{X} ; \widehat{Y} \mid \widehat{Z}=z) \lesssim|\Sigma| \frac{\log (1 / \delta)}{N} \log |\Sigma| \lesssim \frac{|\Sigma|^{2} \log (1 / \delta)}{N}
$$

is even smaller. Rescaling $\delta$, we get with probability $1-\delta$ that

$$
I(\widehat{X} ; \widehat{Y} \mid \widehat{Z})=\sum_{z} \widehat{P}_{z} I(\widehat{X} ; \widehat{Y} \mid \widehat{Z}=z) \lesssim \frac{\log N}{N}|\Sigma|^{3} \log \frac{|\Sigma|}{\delta}
$$

which is at most $\varepsilon$ for the desired $N$.

Proof of (2): Consider the set $S$ of $z \in \sum$ which satisfy $P_{z} \times I(X ; Y \mid$ $\left.Z=z^{*}\right) \geq \frac{I(X ; Y \mid Z)}{2|\Sigma|}$. Note that this implies $P_{z} \geq \frac{\varepsilon}{2|\Sigma| \log |\Sigma|}$. We also have,

$$
\begin{aligned}
& \sum_{z \in S} P_{z} \times I(X ; Y \mid Z=z) \\
& =\sum_{z} P_{z} \times I(X ; Y \mid Z=z)-\sum_{z \notin S} P_{z} \times I(X ; Y \mid Z=z) \\
& \geq I(X ; Y \mid Z)-|\Sigma| \frac{I(X, Y \mid Z)}{2|\Sigma|} \\
& \geq I(X, Y \mid Z) / 2
\end{aligned}
$$


Our $N$ is large enough that for $z \in S, \mathbb{E}\left[N_{z}\right]=P_{z} N \gtrsim \log (|\Sigma| / \delta)$. Hence, with probability $1-\delta$, we have $N_{z} \geq N P_{z} / 2$ for all $z \in S$. Then Corollary 4.6 gives us, with probability $1-\delta$,

$I(\widehat{X} ; \widehat{Y} \mid \widehat{Z}=z) \geq C_{1} I(X ; Y \mid Z=z)-2 C_{2}|\Sigma|^{2} \frac{\log \left(0.5 N P_{z}\right)}{N P_{z}} \log \frac{|\Sigma|}{\delta}$, for all $z \in S$. Multiplying $P_{z}$ and summing over all $z \in S$ give us:

$$
\begin{aligned}
& I(\widehat{X} ; \widehat{Y} \mid \widehat{Z}) \geq \sum_{z \in S} \widehat{P}_{z} I(\widehat{X} ; \widehat{Y} \mid \widehat{Z}=z) \\
& \quad \geq \sum_{z \in S} \frac{P_{z}}{2}\left(C_{1} I(X ; Y \mid Z=z)-2 C_{2}|\Sigma|^{2} \frac{\log \left(0.5 N P_{z}\right)}{N P_{z}} \log \frac{|\Sigma|}{\delta}\right) \\
& \quad \geq \frac{C_{1}}{4} I(X ; Y \mid Z)-C_{2}|\Sigma|^{3} \frac{\log N}{N} \log \frac{|\Sigma|}{\delta} .
\end{aligned}
$$

For $N$ as large as given, the term being subtracted is at most $\frac{\varepsilon C_{1}}{8}$, which is at most half the first term.

A (non-conditional) independence tester with a sample complexity of $|\Sigma|^{-1}$-factor of that of Theorem 1.3 closely follows from the above proof.

\section{TREE STRUCTURE RECOVERY}

\subsection{Non-realizable Case}

Let $P$ be an unknown distribution over $\Sigma^{n}$ and $\hat{P}$ be the empirical distribution of $P$ for a certain number of samples to be fixed later. Our algorithm Chow-Liu returns a maximum spanning tree $\hat{T}$ of the complete graph whose edge weights for every pair of variables are given by the estimated mutual informations with respect to $\hat{P}$.

Let $T^{*}$ be the tree minimizing $D\left(P \| P_{T^{*}}\right)$. Recall that $\operatorname{wt}_{P}(T)$ is defined as the sum of the pairwise mutual informations across $T$. By Corollary 3.4, the Chow-Liu algorithm will return a tree $\widehat{T}$ satisfying

$$
D\left(P \| P_{\widehat{T}}\right) \leq D\left(P \| P_{T^{*}}\right)+\varepsilon
$$

if $\operatorname{wt}_{P}(\widehat{T}) \geq \operatorname{wt}_{P}\left(T^{*}\right)-\varepsilon$. Since $\widehat{T}$ maximizes $\operatorname{wt}_{\widehat{P}}(\widehat{T})$, it would suffice to ensure $\operatorname{wt}_{\widehat{P}}(T)=\operatorname{wt}_{P}(T) \pm \varepsilon / 2$ for all $T$; and therefore it would suffice for

$$
I(\widehat{X} ; \widehat{Y})=I(X ; Y) \pm \frac{\varepsilon}{2 n}
$$

for all pairs of variables $(X, Y)$. The following result is standard, which analyzes the the plug-in estimator $H(\widehat{X})$ for estimating a single discrete entropy $H(X)$ to $\pm \varepsilon$ with probability $1-\delta$.

Theorem $5.1([4,48])$. For $N \gtrsim\left(\frac{|\Sigma|}{\varepsilon}+\frac{1}{\varepsilon^{2}} \log \frac{1}{\delta} \log ^{2}\left(\frac{|\Sigma|}{\varepsilon} \log \frac{1}{\delta}\right)\right)$, $|H(\widehat{X})-H(X)| \leq \varepsilon$ with probability at least $(1-\delta)$.

Since $I(X ; Y)=H(X)+H(Y)-H(X, Y)$, Theorem 5.1 tells us that once

$$
N \gtrsim\left(\frac{|\Sigma|^{2} n}{\varepsilon}+\frac{n^{2}}{\varepsilon^{2}} \log \frac{n}{\delta} \log ^{2}\left(\frac{n|\Sigma|}{\varepsilon} \log \frac{n}{\delta}\right)\right),
$$

all the pairwise mutual informations of the variables of $P$ will be estimated to within $\frac{\varepsilon}{2 n}$. In that case, Chow-Liu would return a tree $T$, the best distribution on which would be close to the closest tree Bayes net of $P$.
Lemma 5.2. Let $P$ be any unknown distribution over $\Sigma^{n}$. Let $Q$ be the tree Bayes net which is closest to $P$ in $\mathrm{KL}$ distance. Then Chow-Liu, when run with $O\left(\frac{|\Sigma|^{2} n}{\varepsilon}+\frac{n^{2}}{\varepsilon^{2}} \log \frac{n}{\delta} \log ^{2}\left(\frac{n|\Sigma|}{\varepsilon} \log \frac{n}{\delta}\right)\right)$ samples, returns a tree $T$ such that there exists a T-structured $R$ with $D(P \| R) \leq$ $D(P \| Q)+\varepsilon$.

We conclude this section by noting that when $P$ itself is a tree Bayes net (realizable case) $D(P \| Q)=0$ and the best Bayes net $R$ on the tree returned by Chow-Liu with the sample complexity of Lemma 5.2 would satisfy $D(P \| R) \leq \varepsilon$ with probability at least (1$\delta)$. In the next section, we show how to bring the sample complexity analysis down from $\widetilde{O}\left(n^{2} / \varepsilon^{2}\right)$ to $\widetilde{O}(n / \varepsilon)$ when $P$ is actually treestructured for some unknown tree.

\subsection{Realizable Case}

We will need the following fact which follows from the chain rule of mutual information.

FACT 5.3. For three random variables $X, Y$, and $Z$

$$
I(X ; Y)-I(X ; Z)=I(X ; Y \mid Z)-I(X ; Z \mid Y) .
$$

Proof. Follows from observing $I(X ; Y)+I(X ; Z \mid Y)=I(X ; Y, Z)$ $=I(X ; Z)+I(X ; Y \mid Z)$.

We also use the following fact about spanning trees:

FACT 5.4. Let $T_{1}$ and $T_{2}$ be two spanning trees on $n$ vertices such that their symmetric difference consists of the edges $E=\left\{e_{1}, e_{2} \ldots, e_{\ell}\right\}$ $\in T_{1} \backslash T_{2}$ and $F=\left\{f_{1}, f_{2} \ldots, f_{\ell}\right\} \in T_{2} \backslash T_{1}$. Then $E$ and $F$ can be paired up, without loss of generality say $\left\langle e_{i}, f_{i}\right\rangle$, such that for all $i$, $T_{1} \cup\left\{f_{i}\right\} \backslash\left\{e_{i}\right\}$ is a spanning tree.

Proof. We use induction on $\ell$. Base case of $\ell=0$ is trivial.

Assume it holds for any two trees $T_{1}$ and $T_{2}$ so that $\left|T_{1} \backslash T_{2}\right|=$ $\left|T_{2} \backslash T_{1}\right|=(l-1)$. Now, pick an arbitrary $e=(u, v) \in T_{1} \backslash T_{2} . T_{1} \backslash\{e\}$ has two connected components, $L \ni u$ and $R=(V \backslash L) \ni v$. In $T_{2}$, there is some path connecting $u$ to $v$. This path starts in $L$ and ends in $R$, so it must have some edge $f$ connecting $L$ to $R$. But $f \notin T_{1}$, since $e \notin T_{2}$ is the only edge connecting $L$ and $R$ in $T_{1}$.

Because $f$ connects $L$ and $R$, which are otherwise unconnected in $T_{1} \backslash\{e\}, T_{1} \cup\{f\} \backslash\{e\}$ is a spanning tree. Thus it is valid to pair $\langle e, f\rangle$. Furthermore, because $f$ lies on the path connecting $e$ in $T_{2}$, $T_{3}:=T_{2} \cup\{e\} \backslash\{f\}$ is also a spanning tree, and it differs in only $\ell-1$ edges from $T_{1}$. Therefore by induction, $T_{3}$ can be paired with $T_{1}$ in the desired way. Adding $\langle e, f\rangle$ to this pairing means that $T_{2}$ can be paired with $T_{1}$.

Theorem 5.5. Let $N$ be such that the bound in Theorem 1.3 holds for a given $\varepsilon, \delta>0$. Then in the realizable case with $n$ nodes, with probability $1-4 n \delta$ Chow-Liu returns a tree $\widehat{T}$ with $D\left(P \| P_{\widehat{T}}\right) \leq \varepsilon n$.

Proof. Let $P$ be the unknown distribution on the true tree $T^{*}$. Let $\widehat{T}$ be the tree returned by the Chow-Liu algorithm with $N$ samples. For any set of vertex pairs $S$, let $\operatorname{wt}(S)$ and $\widehat{\mathrm{wt}}(S)$ denote the sum of mutual information over all pairs in $S$ with respect to the true and empirical distributions respectively, so $\operatorname{wt}\left(T^{*}\right)$ and $\widehat{\mathrm{wt}}(\widehat{T})$ are each maximal over spanning trees. 
For our analysis, we make at most $4 n$ invocations of Theorem 1.3. We will assume the conclusion holds in all cases, as happens with at least $1-4 n \delta$ probability.

Let $\left\{\left\langle e_{i}, f_{i}\right\rangle\right\}_{i}$ be a pairing given by Fact 5.4 for $T^{*}$ and $\widehat{T}$. By (2.1),

$$
D\left(P \| P_{\widehat{T}}\right)=\operatorname{wt}\left(T^{*}\right)-\operatorname{wt}(\widehat{T})=\sum_{i}\left(\operatorname{wt}\left(\left\{e_{i}\right\}\right)-\operatorname{wt}\left(\left\{f_{i}\right\}\right)\right) .
$$

For any $i$, let $e_{i}=\left(X_{i}, Y_{i}\right) \in T^{*}$ and $f_{i}=\left(W_{i}, Z_{i}\right) \in \widehat{T}$. Because $T^{*} \cup\left\{f_{i}\right\} \backslash\left\{e_{i}\right\}$ is a spanning tree, the path connecting $W_{i}$ and $Z_{i}$ in $T^{*}$ must go through $e_{i}$. Without loss of generality let $W \sim X \rightarrow$ $Y \leadsto Z$ be this path (it is possible that $W=X$ or $Y=Z$ ). Hence, from Theorem 3.2, we have for the true distribution

and so

$$
I\left(X_{i} ; Z_{i} \mid Y_{i}\right)=I\left(Z_{i} ; W_{i} \mid X_{i}\right)=0
$$

$$
\begin{aligned}
& I\left(X_{i} ; Y_{i}\right)-I\left(W_{i} ; Z_{i}\right) \\
& =I\left(X_{i} ; Y_{i}\right)-I\left(X_{i} ; Z_{i}\right)+I\left(X_{i} ; Z_{i}\right)-I\left(W_{i} ; Z_{i}\right) \\
& =I\left(X_{i} ; Y_{i} \mid Z_{i}\right)-I\left(X_{i} ; Z_{i} \mid Y_{i}\right)+I\left(X_{i} ; Z_{i} \mid W_{i}\right)-I\left(Z_{i} ; W_{i} \mid X_{i}\right) \\
& =I\left(X_{i} ; Y_{i} \mid Z_{i}\right)+I\left(Z_{i} ; X_{i} \mid W_{i}\right) .
\end{aligned}
$$

On the other hand the empirical distribution will have

$$
\begin{aligned}
& I\left(\widehat{X}_{i} ; \widehat{Y}_{i}\right)-I\left(\widehat{W}_{i} ; \widehat{Z}_{i}\right) \\
& =I\left(\widehat{X}_{i} ; \widehat{Y}_{i} \mid \widehat{Z}_{i}\right)-I\left(\widehat{X}_{i} ; \widehat{Z}_{i} \mid \widehat{Y}_{i}\right)+I\left(\widehat{Z}_{i} ; \widehat{X}_{i} \mid \widehat{W}_{i}\right)-I\left(\widehat{Z}_{i} ; \widehat{W}_{i} \mid \widehat{X}_{i}\right) .
\end{aligned}
$$

Because $\widehat{T}$ is maximal under $\widehat{\mathrm{wt}}$,

$$
\begin{aligned}
0 & \geq \widehat{\mathrm{wt}}\left(T^{*}\right)-\widehat{\mathrm{wt}}(\widehat{T}) \\
= & \sum_{i}\left(I\left(\widehat{X}_{i} ; \widehat{Y}_{i}\right)-I\left(\widehat{W}_{i} ; \widehat{Z}_{i}\right)\right) \\
= & \sum_{i}\left(I\left(\widehat{X}_{i} ; \widehat{Y}_{i} \mid \widehat{Z}_{i}\right)+I\left(\widehat{Z}_{i} ; \widehat{X}_{i} \mid \widehat{W}_{i}\right)\right)- \\
& \quad \sum_{i}\left(I\left(\widehat{X}_{i} ; \widehat{Z}_{i} \mid \widehat{Y}_{i}\right)+I\left(\widehat{Z}_{i} ; \widehat{W}_{i} \mid \widehat{X}_{i}\right)\right) .
\end{aligned}
$$

We invoke Theorem 1.3 with $\varepsilon^{\prime}:=C \varepsilon / 10$ where $C<1$ is the constant given by the theorem. As a consequence of Theorem 1.3 and the fact that each of $I\left(X_{i} ; Z_{i} \mid Y_{i}\right)=I\left(Z_{i} ; W_{i} \mid X_{i}\right)=0$, the second sum is at most $C \varepsilon n / 5$.

On the other hand, Theorem 1.3 implies that

$$
I\left(\widehat{X}_{i} ; \widehat{Y}_{i} \mid \widehat{Z}_{i}\right) \geq C I\left(X_{i} ; Y_{i} \mid Z_{i}\right)-C \varepsilon^{\prime}
$$

and similarly for $I\left(\widehat{Z}_{i} ; \widehat{X}_{i} \mid \widehat{W}_{i}\right)$. As a result, the first sum has

$$
\begin{aligned}
& \sum_{i}\left(I\left(\widehat{X}_{i} ; \widehat{Y}_{i} \mid \widehat{Z}_{i}\right)+I\left(\widehat{Z}_{i} ; \widehat{X}_{i} \mid \widehat{W}_{i}\right)\right) \\
& \quad \geq C \sum_{i}\left(I\left(X_{i} ; Y_{i} \mid Z_{i}\right)+I\left(Z_{i} ; X_{i} \mid W_{i}\right)-2 \varepsilon^{\prime}\right) \\
& \quad \geq C\left(\operatorname{wt}\left(T^{*}\right)-\operatorname{wt}(\widehat{T})\right)-2 C \varepsilon^{\prime} n
\end{aligned}
$$

by (5.1). Combining these bounds into (5.5),

or

$$
0 \geq C\left(\operatorname{wt}\left(T^{*}\right)-\operatorname{wt}(\widehat{T})\right)-\frac{1}{5}\left(C^{2}+C\right) \varepsilon n
$$

$$
D\left(P \| P_{\widehat{T}}\right)=\operatorname{wt}\left(T^{*}\right)-\operatorname{wt}(\widehat{T}) \leq \frac{1}{5}(C+1) \varepsilon n \leq \varepsilon n .
$$

\section{DISTRIBUTION RECOVERY}

This section shows how, for a fixed tree $T$, to find a $T$-structured distribution $Q$ with $D(P \| Q) \leq D\left(P \| P_{T}\right)+\varepsilon$. We start by analyzing how to learn an arbitrary distribution over $\Sigma$.

\subsection{KL Learning of Discrete Distributions}

Given $N$ samples from a distribution $P$ over $\Sigma$, the "add-1" empirical estimator is based on Laplace's rule of succession. This distribution $Q$ is defined by: for each item $i \in \Sigma$, if $i$ appears $T_{i}$ times in the samples, then $Q_{i}=\frac{T_{i}+1}{N+|\Sigma|}$. Kamath, Orlitsky, Pichapati and Suresh [34] have analyzed the expected behavior of the add-1 empirical estimator. In this section, we analyze its behavior in the high-probability regime.

Theorem 6.1. Let $P$ be a distribution over $\Sigma$ and $N \geq 1$. Let $Q$ be the empirical add-1 estimator from $N$ samples of $P$. There is an universal constant $C>0$ such that, with probability $1-\delta$,

$$
D(P \| Q) \leq \frac{C|\Sigma| \log \frac{|\Sigma|}{\delta} \log N}{N} .
$$

Proof. Let $C^{\prime}>1$ be a large constant to be determined later. If $N \leq C^{\prime}|\Sigma|$, the result follows from $D(P \| Q) \leq \log \frac{1}{\min _{i} Q_{i}} \leq$ $\log (N+|\Sigma|) \lesssim \log |\Sigma|$, so we may assume $N \geq C^{\prime}|\Sigma|$. Then

$$
\begin{aligned}
D(P \| Q) & =\sum_{i} P_{i} \log \frac{P_{i}}{Q_{i}} \\
& =\sum_{i} f\left(P_{i}-Q_{i}, Q_{i}\right) \\
& \lesssim \sum_{i} \min \left(\frac{\left(P_{i}-Q_{i}\right)^{2}}{Q_{i}},\left|P_{i}-Q_{i}\right| \log \left(1+\frac{\left|P_{i}-Q_{i}\right|}{Q_{i}}\right)\right) \\
& \lesssim \sum_{i} \min \left(\frac{\left(P_{i}-Q_{i}\right)^{2}}{Q_{i}},\left|P_{i}-Q_{i}\right| \log N\right) .
\end{aligned}
$$

(Since $Q_{i} \geq \frac{1}{N+|\Sigma|}$ )

We also know from Claim 4.4 that with probability at least $1-\delta$ for each $i$,

$$
\begin{aligned}
\left|P_{i}-\frac{T_{i}}{N}\right| & \lesssim \sqrt{\frac{P_{i} \log \frac{1}{\delta}}{N}}+\frac{\log \frac{1}{\delta}}{N} \\
\Longrightarrow\left|P_{i}-Q_{i}\right| & \lesssim \sqrt{\frac{P_{i} \log \frac{1}{\delta}}{N}}+\frac{\log \frac{1}{\delta}}{N}+\left|\frac{T_{i}}{N}-\frac{T_{i}+1}{N+|\Sigma|}\right| \\
& =\sqrt{\frac{P_{i} \log \frac{1}{\delta}}{N}}+\frac{\log \frac{1}{\delta}}{N}+\frac{|| \Sigma\left|T_{i} / N-1\right|}{N+|\Sigma|} \\
& \lesssim \sqrt{\frac{P_{i} \log \frac{1}{\delta}}{N}}+\frac{\log \frac{1}{\delta}}{N}+\frac{|\Sigma|}{N+|\Sigma|} \frac{T_{i}}{N} \\
& \lesssim \sqrt{\frac{P_{i} \log \frac{1}{\delta}}{N}}+\frac{\log \frac{1}{\delta}}{N}
\end{aligned}
$$




$$
\begin{aligned}
& +\frac{|\Sigma|}{N+|\Sigma|}\left(P_{i}+\sqrt{\frac{P_{i} \log \frac{1}{\delta}}{N}}+\frac{\log \frac{1}{\delta}}{N}\right) \\
& \lesssim \sqrt{\frac{P_{i} \log \frac{1}{\delta}}{N}}+\frac{\log \frac{1}{\delta}}{N}+\frac{|\Sigma|}{N+|\Sigma|} P_{i}
\end{aligned}
$$

If $P_{i} \leq \frac{C^{\prime} \log \frac{1}{\delta}}{N}$, then $\left|P_{i}-Q_{i}\right| \log N \lesssim \frac{\log \frac{1}{\delta}}{N} \log N$.

If $P_{i}>\frac{C^{\prime} \log \frac{1}{\delta}}{N}$, then $\left|P_{i}-Q_{i}\right| \lesssim P_{i}\left(\frac{1}{\sqrt{C^{\prime}}}+\frac{1}{C^{\prime}}+\frac{1}{C^{\prime}+1}\right)$ is at most $\frac{P_{i}}{2}$ for sufficiently large $C^{\prime}$, so $Q_{i} \geq P_{i} / 2$ and hence $\frac{\left(P_{i}-Q_{i}\right)^{2}}{Q_{i}} \lesssim$ $\frac{\log \frac{1}{\delta}}{N}+P_{i}\left(\frac{|\Sigma|}{N+|\Sigma|}\right)^{2}$

By a union bound, with probability at least $1-|\Sigma| \delta$ we have that these equations hold for all $i$. If true, then

$$
D(P \| Q) \lesssim \frac{|\Sigma|}{N} \log \frac{1}{\delta} \log N+\left(\frac{|\Sigma|}{N+|\Sigma|}\right)^{2} \lesssim \frac{|\Sigma|}{N} \log \frac{1}{\delta} \log N
$$

Rescaling $\delta$ gives the desired bound.

\subsection{Learning Trees}

We are ready to prove the main result of this section. Algorithm 3 shows the algorithm we analyze below.

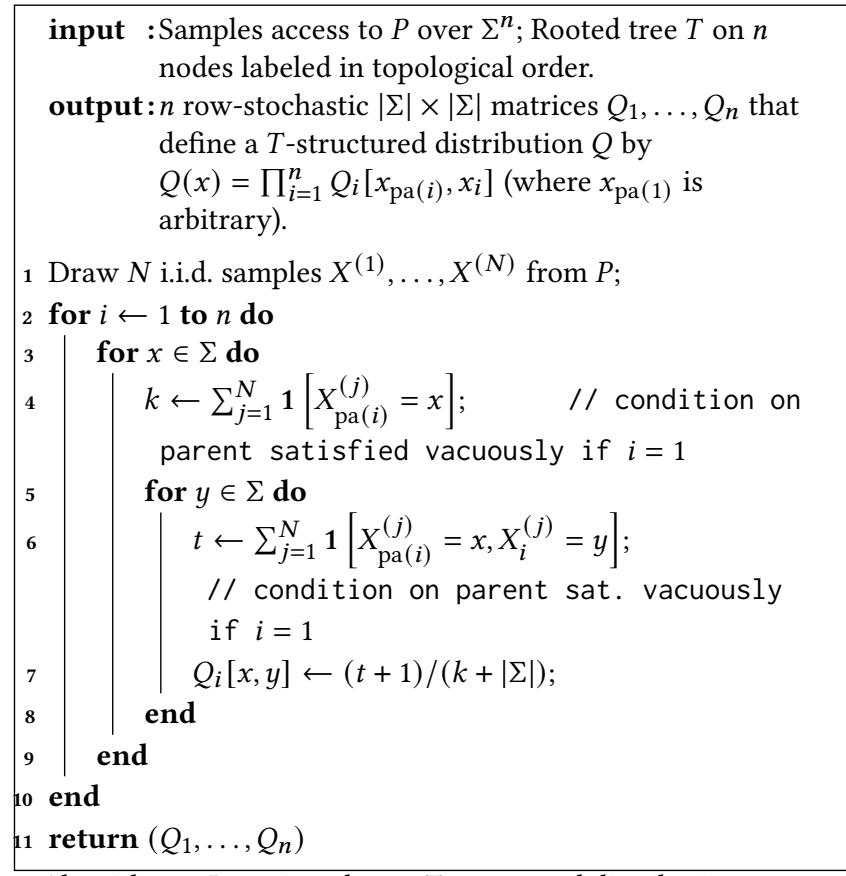

Algorithm 3: Learning closest $T$-structured distribution

Theorem 1.4. Let $P$ be a discrete distribution over $\Sigma^{n}$. Let $T$ be a tree on $n$ vertices, and $Q$ be a T-structured distribution with conditional probabilities at each node estimated using the empirical add-1 estimator on

$$
N=\Theta\left(\frac{n|\Sigma|^{2}}{\varepsilon} \log \frac{n|\Sigma|}{\delta} \log \left(\frac{n|\Sigma|}{\varepsilon} \log \frac{1}{\delta}\right)\right)
$$

samples from $P$. Then

$$
D(P \| Q)-D\left(P \| P_{T}\right) \leq \varepsilon
$$

with probability $1-\delta$.

Proof. Note that $D(P \| Q)$ is bounded, because $Q(x)>0$ for all $x$. By Lemma 3.3 and (2.1), the learned $T$-structured distribution $Q$ satisfies

$$
\begin{aligned}
& D(P \| Q)-D\left(P \| P_{T}\right) \\
& =\sum_{i \in[n]} \sum_{x \in \Sigma} \operatorname{Pr}\left[X_{\mathrm{pa}(i)}=x\right] \cdot D\left(X_{i}\left|X_{\mathrm{pa}(i)}=x \| X_{i}^{\prime}\right| X_{\mathrm{pa}(i)}^{\prime}=x\right)
\end{aligned}
$$

where $X \sim P$ and $X^{\prime} \sim Q$. Now, the node-wise conditional probabilities of $Q$ are the add-1 empirical distribution of the conditional probabilities of $P$. Therefore by Theorem 6.1, if we have $k$ samples of $\left(X \mid X_{\mathrm{pa}(i)}=x\right)$, we will have with probability $1-\delta$ that

$$
\begin{aligned}
D\left(X_{i}\left|X_{\mathrm{pa}(i)}=x \| X_{i}^{\prime}\right| X_{\mathrm{pa}(i)}^{\prime}=x\right) & \lesssim \frac{|\Sigma| \log (|\Sigma| / \delta) \log k}{k} \\
& \leq \frac{|\Sigma| \log (|\Sigma| / \delta) \log N}{k} .
\end{aligned}
$$

If $\mathbb{E}[k]=N \operatorname{Pr}\left[X_{\mathrm{pa}(i)}=x\right]>15 \log \frac{1}{\delta}$, then by a Chernoff bound, with probability $1-\delta$ we have $k>\frac{1}{2} \mathbb{E}[k]$ and

$$
\begin{aligned}
& \operatorname{Pr}\left[X_{\mathrm{pa}(i)}=x\right] \cdot D\left(X_{i}\left|X_{\mathrm{pa}(i)}=x \| X_{i}^{\prime}\right| X_{\mathrm{pa}(i)}^{\prime}=x\right) \\
& \lesssim \frac{|\Sigma| \log (|\Sigma| / \delta) \log N}{N}
\end{aligned}
$$

On the other hand, if $\mathbb{E}[k] \leq 15 \log \frac{1}{\delta}$, then $D\left(X_{i} \mid X_{\mathrm{pa}(i)}=\right.$ $\left.x \| X_{i}^{\prime} \mid X_{\mathrm{pa}(i)}^{\prime}=x\right) \leq \log (k+|\Sigma|)$ [because $Q$ is the add-1 estimator, so the minimum probability is $\left.\frac{1}{k+|\Sigma|}\right]$ and we have

$$
\begin{aligned}
& \operatorname{Pr}\left[X_{\mathrm{pa}(i)}=x\right] \cdot D\left(X_{i}\left|X_{\mathrm{pa}(i)}=x \| X_{i}^{\prime}\right| X_{\mathrm{pa}(i)}^{\prime}=x\right) \\
& \leq \frac{\mathbb{E}[k]}{N} \log (k+|\Sigma|) \\
& \lesssim \frac{\log \frac{1}{\delta} \log N}{N}
\end{aligned}
$$

Regardless, each term in (6.1) is bounded by (6.2). Taking a union bound, with probability $1-n|\Sigma| \delta$ we have

$$
D(P \| Q)-D\left(P \| P_{T}\right) \lesssim n|\Sigma| \frac{|\Sigma| \log (|\Sigma| / \delta) \log N}{N} .
$$

Rescaling $\delta$ and choosing $N$ appropriately gives the result.

The algorithm and analysis in Theorem 1.4 straightforwardly generalizes to Bayes nets. This shows that if $G$ is a directed acyclic graph with in-degree bounded by $d$, we can obtain a $G$-structured distribution $Q$ using $\widetilde{O}\left(n|\Sigma|^{d+1} / \varepsilon\right)$ samples from $P$ which satisfies $D(P \| Q)-D\left(P \| P_{G}\right) \leq \varepsilon$, where $P_{G}=\arg \min _{G \text {-struct. } R} D(P \| R)$.

\section{LOWER BOUNDS FOR STRUCTURE RECOVERY}

\subsection{Non-Realizable Case}

This section focuses on the non-realizable case, i.e., the input distribution is not necessarily a tree structured distribution. We prove 
that $\Omega\left(n^{2} / \varepsilon^{2}\right)$ samples from a distribution $P$ over $\{0,1\}^{n}$ are required to find an $\varepsilon$-approximate tree for $P$. First, we prove a lower bound for $n=3$. We define three distributions $R_{1}, R_{2}, R_{3}$ each over $\{0,1\}^{3}$ as follows.

Let $B_{1} \sim \operatorname{Ber}(1 / 2), B_{2} \sim \operatorname{Ber}(1 / 2)$, and $B_{3} \sim \operatorname{Ber}(1 / 2)$ be three i.i.d. random bits. In $R_{1}: X_{1}, Y_{1}, Z_{1}$ copies $B_{1}$ with probabilities $\frac{3}{4}+$ $\varepsilon, \frac{3}{4}+\varepsilon, \frac{3}{4}-\varepsilon$ respectively and with the remaining probabilities, they independently follow $\operatorname{Ber}(1 / 2)$. In $R_{2}: X_{2}, Y_{2}, Z_{2}$ copies $B_{2}$ with probabilities $\frac{3}{4}+\varepsilon, \frac{3}{4}-\varepsilon, \frac{3}{4}+\varepsilon$ respectively and with the remaining probabilities, they independently follow $\operatorname{Ber}(1 / 2)$. In $R_{3}: X_{3}, Y_{3}, Z_{3}$ copies $B_{3}$ with probabilities $\frac{3}{4}-\varepsilon, \frac{3}{4}+\varepsilon, \frac{3}{4}+\varepsilon$ respectively and with the remaining probabilities, they independently follow $\operatorname{Ber}(1 / 2)$. We need the following Fact to get our lower bound for $n=3$.

FACT 7.1. (i) $D\left(R_{1} \| R_{2}\right)=O\left(\varepsilon^{2}\right)$ and (ii) $I\left(X_{1} ; Y_{1}\right)-I\left(X_{1} ; Z_{1}\right) \geq$ $0.4 \varepsilon$.

Proof. The calculations are skipped.

LEMMA 7.2. (i) Every tree $T$ on 3 vertices is not $0.4 \varepsilon$-approx. for one of $R_{1}, R_{2}, R_{3}$.

(ii) Given samples from a uniformly random distribution among $R_{1}, R_{2}, R_{3}, \Omega\left(1 / \varepsilon^{2}\right)$ samples are needed to rule out an incorrect one with probability at least $4 / 5$.

Observe that Lemma 7.2 implies that if the distribution that generates the samples is chosen uniformly at random among $R_{1}, R_{2}, R_{3}$, then any algorithm that outputs an $\varepsilon$-approximate tree with error probability $<1 / 5$ must draw $\Omega\left(\varepsilon^{-2}\right)$ samples.

Proof of Lemma 7.2. For part (i), note that the possible trees are $G: X-Z-Y, H: X-Y-Z$ and $F: Y-X-Z$. Let $R_{1, G}$ denote the closest $G$-structured distribution to $R$ and so on. Then using Corollary 3.4 and Fact 7.1, $D\left(R \| R_{1, G}\right)-D\left(R \| R_{1, H}\right)=I\left(X_{1} ; Y_{1}\right)-I\left(X_{1} ; Z_{1}\right) \geq$ $0.4 \varepsilon$, which means $D\left(R \| R_{1, G}\right) \geq 0.4 \epsilon$. Similarly for $R_{2}$ and $R_{3}$ by symmetric calculations.

The proof for part (ii) is skipped.

We can now prove the main result of this section.

THEOREM 7.3. The sample complexity of computing an $\varepsilon$-approx. tree on $n$ variables with error probability less than $1 / 3$ is $\Omega\left(n^{2} \varepsilon^{-2}\right)$.

Proof. Suppose $n=3 m$ is a multiple of 3 . We consider the $n$ variables as being divided into $m$ blocks, each of size 3 . Let $P$ be a random distribution on $\{0,1\}^{n}$, defined by setting the distribution of the $i$ 'th block to be $R$ or $R^{\prime}$ with probability $1 / 2$ each independently, where $R$ and $R^{\prime}$ satisfy Lemma 7.2.

For the sake of contradiction, suppose we have an algorithm that draws $c n^{2} \varepsilon^{-2}$ samples from $P$ (for a sufficiently small constant c) and outputs an $\varepsilon$-approximate tree $T$ with probability at least $2 / 3$ (over the choice of $P$ as well as the algorithm's randomness). Since each block is independent, without loss of generality, $T$ is a union of disjoint trees $T_{1}, \ldots, T_{m}$ for each block. By Lemma 7.2 (with $\varepsilon$ replaced with $10 \varepsilon / \mathrm{m}$ ), each $T_{i}$ is not $10 \varepsilon / \mathrm{m}$-approximate with probability at least $1 / 5$. Hence, by a Chernoff bound, with probability $>2 / 3$, for at least $\frac{m}{10}$ trees, $T_{i}$ is not $10 \varepsilon / m$-approximate. Therefore, for any $T$-structured distribution $Q, D(P \| Q)>\frac{m}{10}$. $\frac{10 \varepsilon}{m}=\varepsilon$

\subsection{Realizable Case}

We now show that if $P$ is a tree-structured distribution on $n$ variables, then $\Omega\left(n \varepsilon^{-1} \log \frac{n}{\varepsilon}\right)$ samples are required to find an $\varepsilon$-approx. tree. As with the non-realizable case, we first show the construction for $n=3$. We define three distributions $R_{1}, R_{2}, R_{3}$ each over $\{0,1\}^{3}$ as follows.

In $R_{1},\left(Y_{1}, Z_{1}\right)$ randomly takes values between $(0,0)$ and $(1,1)$, and $X_{1}$ copies the other 2 bits with $(1-\varepsilon)$ probability and with the remaining probability follows $\operatorname{Ber}(1 / 2) . R_{2}$ and $R_{3}$ are defined symmetrically with the restrictions $X_{2}=Z_{2}$ and $X_{3}=Y_{3}$ respectively. Let $H(P, Q)=\sqrt{\frac{1}{2} \sum_{x \in \Omega}(\sqrt{P(x)}-\sqrt{Q(x)})^{2}}$ be the Hellinger distance between two distributions. We need the following facts.

FACT 7.4. (i) $H^{2}\left(R_{1}, R_{2}\right)=\Theta(\varepsilon)$ and (ii) $I\left(Y_{1} ; Z_{1}\right)-I\left(X_{1} ; Z_{1}\right)=$ $\Theta\left(\varepsilon \log \frac{1}{\varepsilon}\right)$.

Proof. The calculations are skipped.

Lemma 7.5. (i) Every tree $T$ on 3 vertices is not $\Theta\left(\varepsilon \log \frac{1}{\varepsilon}\right)$ approximate for one of $R_{1}, R_{2}, R_{3}$.

(ii) Given samples from a random distribution from $R_{1}, R_{2}, R_{3}$, $\Omega(1 / \varepsilon)$ samples are needed to rule out an incorrect one with $4 / 5$ probability.

The same argument used for Theorem 7.3 implies:

THEOREM 7.6. The sample complexity of computing an $\varepsilon$-approx. tree for a tree-structured distribution on $n$ variables with error probability less than $1 / 3$ is $\Omega\left(n \varepsilon^{-1} \log \frac{n}{\varepsilon}\right)$.

Proof of Lemma 7.5. For part (i), let $G:=X-Y-Z$ and $H:=$ $Y-X-Z$. Let $R_{1, H}$ be the closest $H$-structured distribution to $R_{1}$ and so on. Then by Corollary 3.4, $D\left(R_{1} \| R_{1, H}\right)-D\left(R_{1} \| R_{1, G}\right)=$ $I\left(Y_{1} ; Z_{1}\right)-I\left(X_{1} ; Z_{1}\right)=\Omega\left(\varepsilon \log \frac{1}{\varepsilon}\right)$ using Fact 7.4. Hence $D\left(R_{1} \| R_{1, H}\right)$ $=\Omega\left(\varepsilon \log \frac{1}{\varepsilon}\right)$. Similarly, it can be shown for the other two trees.

The proof for part (ii) is skipped.

\section{ACKNOWLEDGMENTS}

Arnab and Sutanu thank Rishi Gajjala for contributing to related discussions and Sanjoy Dasgupta for clarifying some questions about [21]. Also, Eric thanks Alex Dimakis for pointing us to [25]. We thank the anonymous reviewers of STOC ' 21 for valuable comments. Arnab was supported in part by NRF-AI Fellowship R-252-100-B13281 and an Amazon Research Award. Sutanu was supported in part by NRF-AI Fellowship R-252-100-B13-281. Eric was supported in part by NSF Award CCF-1751040 (CAREER). Vinod was supported in part by NSF CCF-184908 and NSF HDR:TRIPODS-1934884 awards.

\section{REFERENCES}

[1] Pieter Abbeel, Daphne Koller, and Andrew Y Ng. 2006. Learning factor graphs in polynomial time and sample complexity. Journal of Machine Learning Research 7 , Aug (2006), 1743-1788.

[2] Jayadev Acharya, Constantinos Daskalakis, and Gautam Kamath. 2015. Optimal Testing for Properties of Distributions. In Advances in Neural Information Processing Systems.

[3] Anima Anandkumar, Daniel J Hsu, Furong Huang, and Sham M Kakade. 2012. Learning mixtures of tree graphical models. In Advances in Neural Information Processing Systems. 1052-1060. 
[4] András Antos and Ioannis Kontoyiannis. 2001. Convergence properties of functional estimates for discrete distributions. Random Structures \& Algorithms 19 , 3-4 (2001), 163-193.

[5] Tugkan Batu, Lance Fortnow, Eldar Fischer, Ravi Kumar, Ronitt Rubinfeld, and Patrick White. 2001. Testing Random Variables for Independence and Identity. In 42nd Annual Symposium on Foundations of Computer Science.

[6] Arnab Bhattacharyya, Sutanu Gayen, Kuldeep S. Meel, and N. V. Vinodchandran. 2020. Efficient Distance Approximation for Structured High-Dimensional Distributions via Learning. CoRR abs/2002.05378 (2020). arXiv:2002.05378 https://arxiv.org/abs/2002.05378

[7] Guy Bresler. 2015. Efficiently learning Ising models on arbitrary graphs. In Proceedings of the forty-seventh annual ACM symposium on Theory of computing. 771-782.

[8] Guy Bresler and Mina Karzand. 2020. Learning a tree-structured Ising model in order to make predictions. Ann. Statist. 48, 2 (04 2020), 713-737. https: //doi.org/10.1214/19-AOS1808

[9] G. Bresler and M. Karzand. 2020. Minimax Prediction in Tree Ising Models In 2020 IEEE International Symposium on Information Theory (ISIT). 1325-1330. https://doi.org/10.1109/ISIT44484.2020.9174341

[10] Guy Bresler, Elchanan Mossel, and Allan Sly. 2013. Reconstruction of Markov random fields from samples: Some observations and algorithms. SIAM f. Comput. 42, 2 (2013), 563-578.

[11] Johannes Brustle, Yang Cai, and Constantinos Daskalakis. 2020. Multi-item mechanisms without item-independence: Learnability via robustness. In Proceedings of the 21st ACM Conference on Economics and Computation. 715-761.

[12] Clément L. Canonne. 2015. A Survey on Distribution Testing: Your Data is Big But is it Blue? Electronic Colloquium on Computational Complexity (ECCC) 22 (2015), 63. http://eccc.hpi-web.de/report/2015/063

[13] Clément L. Canonne. 2020. Personal communication.

[14] Clément L. Canonne, Ilias Diakonikolas, Daniel M. Kane, and Alistair Stewart. 2018. Testing conditional independence of discrete distributions. In Proceedings of the 50th Annual ACM SIGACT Symposium on Theory of Computing, STOC 2018 ACM, 735-748. https://doi.org/10.1145/3188745.3188756

[15] Clément L. Canonne, Ilias Diakonikolas, Daniel M. Kane, and Alistair Stewart. 2020. Testing Bayesian Networks. IEEE Trans. Inf. Theory 66, 5 (2020), 3132-3170. https://doi.org/10.1109/TIT.2020.2971625

[16] Anton Chechetka and Carlos Guestrin. 2008. Efficient principled learning of thin junction trees. In Advances in Neural Information Processing Systems. 273-280.

[17] David Maxwell Chickering. 1995. Learning Bayesian Networks is NP-Complete In Learning from Data - Fifth International Workshop on Artificial Intelligence and Statistics, AISTATS, Doug Fisher and Hans-Joachim Lenz (Eds.).

[18] C Chow and T Wagner. 1973. Consistency of an estimate of tree-dependent probability distributions. IEEE Transactions on Information Theory 19, 3 (1973), 369-371.

[19] C. K. Chow and C. N. Liu. 1968. Approximating discrete probability distributions with dependence trees. IEEE Trans. Inf. Theory 14, 3 (1968), 462-467. https: //doi.org/10.1109/TIT.1968.1054142

[20] Paul Dagum and Michael Luby. 1997. An optimal approximation algorithm for Bayesian inference. Artificial Intelligence 93, 1 (1997), 1-28.

[21] Sanjoy Dasgupta. 1997. The Sample Complexity of Learning Fixed-Structure Bayesian Networks. Mach. Learn. 29, 2-3 (1997), 165-180. https://doi.org/10 1023/A:1007417612269

[22] Sanjoy Dasgupta. 2013. Learning Polytrees. CoRR abs/1301.6688 (2013) arXiv:1301.6688 http://arxiv.org/abs/1301.6688

[23] Constantinos Daskalakis, Nishanth Dikkala, and Gautam Kamath. 2019. Testing Ising Models. IEEE Trans. Inf. Theory 65, 11 (2019), 6829-6852. https://doi.org/ 10.1109/TIT.2019.2932255

[24] Constantinos Daskalakis and Qinxuan Pan. 2017. Square Hellinger Subadditivity for Bayesian Networks and its Applications to Identity Testing. In Proceedings of the 30th Conference on Learning Theory, COLT, Satyen Kale and Ohad Shamir (Eds.).

[25] Constantinos Daskalakis and Qinxuan Pan. 2020. Tree-structured Ising models can be learned efficiently. CoRR abs/2010.14864 (2020). arXiv:2010.14864 https: //arxiv.org/abs/2010.14864

[26] Luc Devroye, Abbas Mehrabian, and Tommy Reddad. 2020. The minimax learning rates of normal and Ising undirected graphical models. Electronic fournal of Statistics 14, 1 (2020), 2338-2361.

[27] Ilias Diakonikolas, Themis Gouleakis, Daniel M. Kane, John Peebles, and Eric Price. 2021. Optimal Testing of Discrete Distributions with High Probability. STOC (2021). arXiv:2009.06540 [cs.DS]

[28] Ilias Diakonikolas, Themis Gouleakis, John Peebles, and Eric Price. 2018. Sampleoptimal identity testing with high probability. In 45th International Colloquium on Automata, Languages, and Programming (ICALP 2018). Schloss Dagstuhl-LeibnizZentrum fuer Informatik.

[29] Ilias Diakonikolas and Daniel M. Kane. 2016. A New Approach for Testing Properties of Discrete Distributions. In IEEE 57th Annual Symposium on Foundations of Computer Science, FOCS, Irit Dinur (Ed.).
[30] Surbhi Goel. 2020. Learning Ising and Potts Models with Latent Variables (Proceedings of Machine Learning Research, Vol. 108), Silvia Chiappa and Roberto Calandra (Eds.). PMLR, Online, 3557-3566.

[31] Oded Goldreich. 2017. Introduction to Property Testing. Cambridge University Press. https://doi.org/10.1017/9781108135252

[32] Oded Goldreich and Dana Ron. 2011. On testing expansion in bounded-degree graphs. In Studies in Complexity and Cryptography. Miscellanea on the Interplay between Randomness and Computation. Springer, 68-75.

[33] Klaus-U Höffgen. 1993. Learning and robust learning of product distributions. In Proceedings of the sixth annual conference on Computational learning theory. 77-83.

[34] Sudeep Kamath, Alon Orlitsky, Dheeraj Pichapati, and Ananda Theertha Suresh. 2015. On Learning Distributions from their Samples. In Proceedings of The 28th Conference on Learning Theory, COLT 2015.

[35] David R. Karger and Nathan Srebro. 2001. Learning Markov networks: maximum bounded tree-width graphs. In Proceedings of the Twelfth Annual Symposium on Discrete Algorithms, January 7-9, 2001, Washington, DC, USA, S. Rao Kosaraju (Ed.). ACM/SIAM, 392-401. http://dl.acm.org/citation.cfm?id=365411.365486

[36] Michael J Kearns and Robert E Schapire. 1994. Efficient distribution-free learning of probabilistic concepts. F. Comput. System Sci. 48, 3 (1994), 464-497.

[37] Michael J Kearns, Robert E Schapire, and Linda M Sellie. 1994. Toward efficient agnostic learning. Machine Learning 17, 2-3 (1994), 115-141.

[38] Adam R. Klivans and Raghu Meka. 2017. Learning Graphical Models Using Multiplicative Weights. In 58th IEEE Annual Symposium on Foundations of Computer Science, FOCS, Chris Umans (Ed.).

[39] Daphne Koller and Nir Friedman. 2009. Probabilistic graphical models: principles and techniques. MIT press.

[40] Frank R Kschischang, Brendan J Frey, and H-A Loeliger. 2001. Factor graphs and the sum-product algorithm. IEEE Transactions on information theory 47, 2 (2001), 498-519.

[41] PS Laplace. 1995. Philosophical Essays on Probabilities, from 5th French edition published 1825, translated AI Dale.

[42] Steffen L Lauritzen. 1996. Graphical models. Vol. 17. Clarendon Press.

[43] Han Liu, Min Xu, Haijie Gu, Anupam Gupta, John Lafferty, and Larry Wasserman. 2011. Forest density estimation. The fournal of Machine Learning Research 12 (2011), 907-951.

[44] Christopher Meek. 2001. Finding a path is harder than finding a tree. Fournal of Artificial Intelligence Research 15 (2001), 383-389.

[45] Marina Meila. 1999. An Accelerated Chow and Liu Algorithm: Fitting Tree Distributions to High-Dimensional Sparse Data. In Proceedings of the Sixteenth International Conference on Machine Learning (ICML 1999), Bled, Slovenia, June 27 - 30, 1999, Ivan Bratko and Saso Dzeroski (Eds.). Morgan Kaufmann, 249-257.

[46] Marina Meila and Michael I Jordan. 2000. Learning with mixtures of trees. Fournal of Machine Learning Research 1, Oct (2000), 1-48.

[47] Mukund Narasimhan and Jeff Bilmes. 2004. PAC-learning bounded tree-width graphical models. In Proceedings of the 20th conference on Uncertainty in artificial intelligence. 410-417.

[48] Liam Paninski. 2003. Estimation of entropy and mutual information. Neural computation 15, 6 (2003), 1191-1253.

[49] Ronitt Rubinfeld. 2012. Taming big probability distributions. ACM Crossroads 19, 1 (2012), 24-28. https://doi.org/10.1145/2331042.2331052

[50] Nathan Srebro. 2003. Maximum likelihood bounded tree-width Markov networks. Artificial intelligence 143, 1 (2003), 123-138.

[51] Vincent YF Tan, Animashree Anandkumar, Lang Tong, and Alan S Willsky. 2011. A large-deviation analysis of the maximum-likelihood learning of Markov tree structures. IEEE Transactions on Information Theory 57, 3 (2011), 1714-1735.

[52] Anshoo Tandon, Vincent YF Tan, and Shiyao Zhu. 2020. Exact Asymptotics for Learning Tree-Structured Graphical Models with Side Information: Noiseless and Noisy Samples. arXiv preprint arXiv:2005.04354 (2020).

[53] Leslie G Valiant. 1984. A theory of the learnable. Commun. ACM 27, 11 (1984), 1134-1142.

[54] Thomas Verma and Judea Pearl. 1990. Equivalence and synthesis of causal models. In UAI '90: Proceedings of the Sixth Annual Conference on Uncertainty in Artificial Intelligence, 1990. Elsevier, 255-270. https://dslpitt.org/uai/displayArticleDetails. jsp? $\mathrm{mmnu}=1 \&$ smnu $=2 \&$ article _id $=1918 \&$ proceeding id $=1006$

[55] Martin J Wainwright. 2006. Estimating the "Wrong" Graphical Model: Benefits in the Computation-Limited Setting. Fournal of Machine Learning Research 7, Sep (2006), 1829-1859.

[56] Martin J Wainwright and Michael Irwin Jordan. 2008. Graphical models, exponential families, and variational inference. Now Publishers Inc.

[57] Rui Wu, R Srikant, and Jian Ni. 2013. Learning loosely connected Markov random fields. Stochastic Systems 3, 2 (2013), 362-404

[58] Shanshan Wu, Sujay Sanghavi, and Alexandros G Dimakis. 2019. Sparse logistic regression learns all discrete pairwise graphical models. In Advances in Neural Information Processing Systems. 8071-8081. 